\title{
Enantioselective Friedel-Crafts Alkylation of Indoles with Nitroalkenes Catalyzed by Bifunctional Tridentate Bis(oxazoline)-Zn(II) Complex
}

\author{
Shao-Feng Lu, Da-Ming Du, ${ }^{*} \quad$ Jiaxi Xu \\ Beijing National Laboratory for Molecular Sciences (BNLMS), Key Laboratory of Bioorganic Chemistry \\ and Molecular Engineering, College of Chemistry and Molecular Engineering, Peking University, \\ Beijing 100871, P. R. China \\ dudm@pku.edu.cn
}

Supporting Information

\section{Contents}

General remarks.

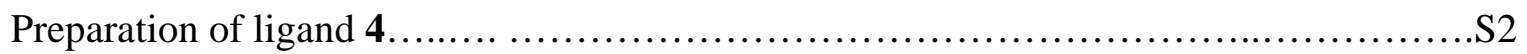

General procedure for the catalytic enantioselective Friedel-Crafts reaction...............S3

Characterization of nitroalkylated indoles.......................................S3

Reference..........................................................................

Copies of HPLC profiles of nitroalkylated indoles..............................S

Copies of ${ }^{1} \mathrm{H}$ NMR and ${ }^{13} \mathrm{C}$ NMR of nitroalkylated indoles $\mathbf{7 g}, \mathbf{7 j}, \mathbf{7 k}, \mathbf{7 l}$ and $\mathbf{7 m} \ldots \ldots \ldots$...S21

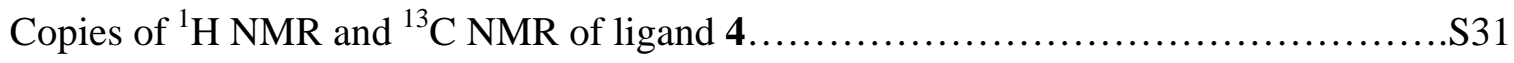




\section{General remarks}

Commercially available compounds were used without further purification. Solvents were dried according to standard procedures. Column chromatography was carried out using silica gel (200-300 mesh). Melting points were measured on an XT-4 melting point apparatus and uncorrected. The ${ }^{1} \mathrm{H}$ NMR spectra were recorded on Mercury 300 and Brucker $400 \mathrm{MHz}$ spectrometers, while the ${ }^{13} \mathrm{C}$ NMR spectra were recorded at 75 and $100 \mathrm{MHz}$ respectively. Elemental analyses were carried out on an Elementar Vario EL instrument. Infrared spectra were obtained on a Nicolet AVATAR 330 FT-IR spectrometer. Mass spectra were obtained on a VG-ZAB-HS (EI) mass spectrometer. Optical rotations were measured on a Perkin-Elmer 341 LC spectrometer. The enatiomeric excess (ee) of the products was determined by chiral HPLC (2-propanol/hexane as the eluent). The nitroalkenes are commercial available or prepared according to literature. ${ }^{1}$

\section{Experimental Procedures}

\section{Preparation of ligand 4}

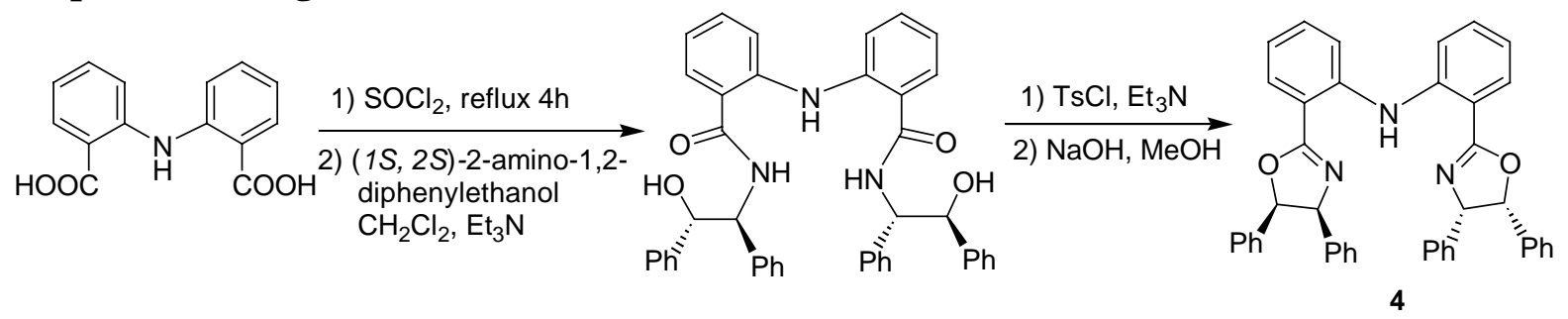

A solution of 2,2'-dicarboxyl diphenylamine $(1.837 \mathrm{~g}, 7.0 \mathrm{mmol})$ and thionyl chloride $(10 \mathrm{~mL})$ was refluxed for $4 \mathrm{~h}$. The excess $\mathrm{SOCl}_{2}$ was removed under reduced pressure to afford the diacyl dichloride. The above diacyl dichloride in $\mathrm{CH}_{2} \mathrm{Cl}_{2}(70 \mathrm{~mL})$ was added dropwise to a solution of $(1 S, 2 S)$-2-amino-1,2-diphenylethanol (2.986 g, $14.0 \mathrm{mmol})$ and $\mathrm{Et}_{3} \mathrm{~N}(4.9 \mathrm{~mL}$, $35.0 \mathrm{mmol})$ in $\mathrm{CH}_{2} \mathrm{Cl}_{2}(20 \mathrm{~mL})$ at $0{ }^{\circ} \mathrm{C}$ and stirred at room temperature for $24 \mathrm{~h}$. The reaction mixture was successively washed with saturated $\mathrm{NH}_{4} \mathrm{Cl}(\mathrm{aq}), \mathrm{HCl}(1 \mathrm{~N})$, saturated $\mathrm{NaHCO}_{3}$ (aq) and brine. The organic layer was dried over anhydrous $\mathrm{Na}_{2} \mathrm{SO}_{4}$, concentrated and purified by silica gel column chromatography using ethyl acetate as the eluting solvent to afford the bis(hydroxyamide) in 67\% yield. To an ice-cold solution of the bis(hydroxyamide) (1.192 g, $1.84 \mathrm{mmol})$ and $\mathrm{Et}_{3} \mathrm{~N}(1.1 \mathrm{~mL}, 8.07 \mathrm{mmol})$ in $\mathrm{CH}_{2} \mathrm{Cl}_{2}(15 \mathrm{~mL})$ was added methanesulfonyl chloride $(0.32 \mathrm{~mL}, 4.04 \mathrm{mmol})$ via syringe. The reaction mixture was allowed to warm to room temperature and stirred for $3 \mathrm{~h}$. Then saturated $\mathrm{NH}_{4} \mathrm{Cl}$ solution (30 $\mathrm{mL}$ ) was poured into the reaction mixture. The organic layer was separated and the aqueous layer was extracted with $\mathrm{CH}_{2} \mathrm{Cl}_{2}(3 \times 15 \mathrm{~mL})$. The combined extracts were washed with brine, dried over anhydrous $\mathrm{Na}_{2} \mathrm{SO}_{4}$, and concentrated in vacuo to afford the crude bismesylate. The crude bismesylate was dissolved in methanol $(4.4 \mathrm{~mL})$ and a solution of $0.162 \mathrm{~g} \mathrm{NaOH}$ in water $(4.4 \mathrm{~mL})$ was added to it. The reaction mixture was refluxed for $4 \mathrm{~h}$ and cooled to room temperature. The methanol was removed under reduced pressure and the residue was extracted with $\mathrm{CH}_{2} \mathrm{Cl}_{2}(3 \times 15 \mathrm{~mL})$. The combined organic layer was washed with brine, 
dried over anhydrous $\mathrm{Na}_{2} \mathrm{SO}_{4}$, and concentrated in vacuo to give the crude product. Purification by column chromatography on silica gel using petroleum ether-ethyl acetate 10:1 as the eluent to afford $700 \mathrm{mg}$ (62\% yield) of 4 as a pale yellow solid. m.p. $175-177{ }^{\circ} \mathrm{C}$. $[\alpha]_{\mathrm{D}}{ }^{20}=+114\left(c 0.67, \mathrm{CH}_{2} \mathrm{Cl}_{2}\right) .{ }^{1} \mathrm{H}$ NMR $\left(400 \mathrm{MHz}, \mathrm{CDCl}_{3}\right): \delta=11.28$ (s, $\left.1 \mathrm{H}\right), 7.96$ (dd, $J=$ 7.8, 1.6 Hz, 2H), 7.60 (d, $J=8.2 \mathrm{~Hz}, 2 \mathrm{H}), 7.38-7.32$ (m, 2H), 7.29-7.27 (m, 6H), 7.18-7.14 (m, 10H), 7.06-7.03 (m, 4H), 6.96-6.93 (m, 2H), 5.08 (d, $J=7.6 \mathrm{~Hz}, 2 \mathrm{H}), 4.92$ (d, $J=7.6$ $\mathrm{Hz}, 2 \mathrm{H}) .{ }^{13} \mathrm{C}$ NMR $\left(100 \mathrm{MHz}, \mathrm{CDCl}_{3}\right): \delta=163.3,143.4,142.0,140.4,131.6,130.6,128.6$, 128.4, 128.1, 127.2, 126.4, 125.7, 119.9, 118.4, 115.7, 87.7, 78.7. IR (neat): 3061, 3030, 1641, 1580, 1518, 1455, 1269, 1052, 762, 747, $698 \mathrm{~cm}^{-1}$. MS (70 eV, EI): $\mathrm{m} / z(\%) 611\left(\mathrm{M}^{+}, 100\right)$, 504 (22), 444 (22), 309 (33), 282 (20), 249 (15), 206 (30), 179 (32), 105 (11). Anal. Calcd for $\mathrm{C}_{42} \mathrm{H}_{33} \mathrm{~N}_{3} \mathrm{O}_{2}$ : C, 82.46; H, 5.44; N, 6.87. Found: C, 82.32; H, 5.56; N, 6.82.

General Procedure for the Catalytic Enantioselective Friedel-Crafts reaction. (R)-3-(2-Nitro-1-phenylethyl)-1H-indole (7a). To a dried Schlenk tube were added<smiles>O=[N+]([O-])CC(c1ccccc1)c1c[nH]c2ccccc12</smiles>
$\mathrm{Zn}(\mathrm{OTf})_{2}$ (9.3 $\left.\mathrm{mg}, 0.025 \mathrm{mmol}\right)$ and ligand 4 (15.3 $\left.\mathrm{mg}, 0.025 \mathrm{mmol}\right)$ under nitrogen followed by addition of the toluene $(2 \mathrm{~mL})$. The solution was stirred at room temperature for $30 \mathrm{~min}$ and the trans- $\beta$-nitrostyrene 6a (74.5 mg, $0.5 \mathrm{mmol}$ ) was added. The mixture was stirred for $10 \mathrm{~min}$ and cooled to $-20{ }^{\circ} \mathrm{C}$ before the indole $5 \mathrm{a}$ (57 $\mathrm{mg}, 0.5 \mathrm{mmol}$ ) was added. After stirring for $15 \mathrm{~h}$ at $-20{ }^{\circ} \mathrm{C}$, the solvent was removed under vacuum. Purification by column chromatography (20\% AcOEt in petroleum ether) afforded the desired product 3-(2-nitro-1-phenylethyl)-1H-indole 7a (125.4 mg, 95\% yield) as an oil. The ee was determined by chiral HPLC on Daicel Chiracel OD-H column (hexane: 2-propanol 70:30, 0.9 $\left.\mathrm{mL} / \mathrm{min}, \mathrm{t}_{\text {minor }}=20.7 \mathrm{~min}, \mathrm{t}_{\text {major }}=24.7 \mathrm{~min}\right) .[\alpha]_{\mathrm{D}}{ }^{20}=-21.3\left(c 0.54, \mathrm{CH}_{2} \mathrm{Cl}_{2}, 94 \%\right.$ ee $) .{ }^{1} \mathrm{H}$ NMR (300 MHz, CDCl $\left.)_{3}\right): \delta=8.02(\mathrm{~s}, 1 \mathrm{H}), 7.43(\mathrm{~d}, J=7.8 \mathrm{~Hz}, 1 \mathrm{H}) 7.31-7.15(\mathrm{~m}, 7 \mathrm{H}), 7.06$ (t, $J=7.6 \mathrm{~Hz}, 1 \mathrm{H}), 6.95$ (d, $J=2.4 \mathrm{~Hz}, 1 \mathrm{H}), 5.16$ (t, $J=8.1 \mathrm{~Hz}, 1 \mathrm{H}), 5.02$ (dd, $J=12.4,7.6$ $\mathrm{Hz}, 1 \mathrm{H}), 4.90$ (dd, $J=12.4,8.6 \mathrm{~Hz}, 1 \mathrm{H}) .{ }^{13} \mathrm{C} \mathrm{NMR}\left(75 \mathrm{MHz}, \mathrm{CDCl}_{3}\right.$ ): $\delta=139.1,136.4$, $128.9,127.7,127.5,126.0,122.6,121.6,119.9,118.8,114.2,111.4,79.5,41.5$. $^{2}{ }^{2}[\alpha]_{\mathrm{D}}{ }^{20}=$ $+25.3\left(c 0.9, \mathrm{CH}_{2} \mathrm{Cl}_{2}, 84 \%\right.$ ee) for $(S)$ configuration.

5-Methoxy-3-[2-nitro-1-phenylethyl]-1H-indole (7b). Prepared according to the general<smiles>COc1ccc2[nH]cc(C(C[N+](=O)[O-])c3ccccc3)c2c1</smiles>
procedure using trans- $\beta$-nitrostyrene $6 \mathbf{6 a}(74.5 \mathrm{mg}, 0.50 \mathrm{mmol})$ and 5-methoxyindole $5 \mathbf{b}$ (73.6 mg, $0.5 \mathrm{mmol})$. Purified by column chromatography (20\% AcOEt in petroleum ether) to yield $142.2 \mathrm{mg}$ (96\%) of $\mathbf{7 b}$ as an oil. The ee was determined by chiral HPLC on Daicel Chiracel OD-H column (hexane: 2-propanol 85:15, 1.0 $\left.\mathrm{mL} / \mathrm{min}, \mathrm{t}_{\text {major }}=27.7 \mathrm{~min}, \mathrm{t}_{\text {minor }}=33.5 \mathrm{~min}\right) .[\alpha]_{\mathrm{D}}{ }^{20}=+33.1\left(\right.$ c $1.15, \mathrm{CH}_{2} \mathrm{Cl}_{2}, 98 \%$ ee $) .{ }^{1} \mathrm{H}$ NMR (300 MHz, CDCl 3 ): $\delta=7.99$ (s, 1H), 7.34-7.19 (m, 6H), 6.96 (d, $J=2.4 \mathrm{~Hz}, 1 \mathrm{H}), 6.86$ (d, $J=2.4 \mathrm{~Hz}, 1 \mathrm{H}), 6.83$ (d, $J=1.5 \mathrm{~Hz}, 1 \mathrm{H}), 5.12$ (t, $J=8.0 \mathrm{~Hz}, 1 \mathrm{H}), 5.02$ (dd, $J=12.3,7.5$ $\mathrm{Hz}, 1 \mathrm{H}), 4.91$ (dd, $J=12.3,8.1 \mathrm{~Hz}, 1 \mathrm{H}), 3.76$ (s, 3H). ${ }^{13} \mathrm{C}$ NMR $\left(75 \mathrm{MHz}, \mathrm{CDCl}_{3}\right): \delta=154.1$, 139.1, 131.5, 128.9, 127.7, 127.5, 126.5, 122.2, 114.0, 112.6, 112.1, 100.7, 79.4, 55.8, 41.5. 
Lit. $^{2}[\alpha]_{\mathrm{D}}^{20}=-25.6\left(c 1.1, \mathrm{CH}_{2} \mathrm{Cl}_{2}, 79 \%\right.$ ee $)$.

5-Chloro-3-[2-nitro-1-phenylethyl]-1H-indole (7c). Prepared according to the general<smiles>O=[N+]([O-])CC(c1ccccc1)c1c[nH]c2ccc(Cl)cc12</smiles>
procedure using trans- $\beta$-nitrostyrene $6 \mathbf{6 a}(74.5 \mathrm{mg}, 0.50 \mathrm{mmol})$ and 5-chloroindole 5c (75.8 mg, $0.5 \mathrm{mmol})$. Purified by column chromatography (20\% AcOEt in petroleum ether) to yield $132.4 \mathrm{mg}$ (88\%) of $7 \mathrm{c}$ as an oil. The ee was determined by chiral HPLC on Daicel Chiracel OD-H column (hexane: 2-propanol 70:30, 0.9 $\left.\mathrm{mL} / \mathrm{min}, \mathrm{t}_{\text {major }}=13.6 \mathrm{~min}, \mathrm{t}_{\text {minor }}=14.8 \mathrm{~min}\right) .[\alpha]_{\mathrm{D}}{ }^{20}=+26.8\left(\right.$ c $1.11, \mathrm{CH}_{2} \mathrm{Cl}_{2}, 81 \%$ ee $) .{ }^{1} \mathrm{H}$ NMR (300 MHz, $\left.\mathrm{CDCl}_{3}\right): \delta=8.12(\mathrm{~s}, 1 \mathrm{H}), 7.37$ (d, $\left.J=2.1 \mathrm{~Hz}, 1 \mathrm{H}\right), 7.35-7.21(\mathrm{~m}, 6 \mathrm{H}), 7.12$ (dd, $J=8.6,2.0 \mathrm{~Hz}, 1 \mathrm{H}), 7.05$ (d, $J=2.4 \mathrm{~Hz}, 1 \mathrm{H}), 5.11$ (t, $J=8.0 \mathrm{~Hz}, 1 \mathrm{H}), 5.01$ (dd, $J=12.3$, $8.1 \mathrm{~Hz}, 1 \mathrm{H}), 4.90$ (dd, $J=12.3,8.1 \mathrm{~Hz}, 1 \mathrm{H}) .{ }^{13} \mathrm{C} \mathrm{NMR}\left(75 \mathrm{MHz} \mathrm{CDCl}_{3}\right): \delta=138.7,134.7$, $129.0,127.7,127.6,127.1,125.6,123.0,122.8,118.3,114.0,112.4,79.3,41.3$. $^{2}{ }^{3}{ }^{3}[\alpha]_{\mathrm{D}}{ }^{\mathrm{rt}}=$ +18 ( $0.370, \mathrm{CHCl}_{3}, 71 \%$ ee).

(R)-1-Methyl-3-[2-nitro1-1-phenylethyl]-1H-indole (7d). Prepared according to the general $\mathrm{Ph} \quad$ procedure using trans- $\beta$-nitrostyrene 6a $(74.5 \mathrm{mg}, 0.50 \mathrm{mmol})$ and 1-methylindole 7d (65.6 mg, 0.5mmol). Purified by column chromatography (20\% AcOEt in petroleum ether) to yield $130.0 \mathrm{mg}$ (93\%) of $\mathbf{4 a}$ as an oil. The ee was determined by chiral HPLC on Daicel Chiracel OD-H column (hexane: 2-propanol 85:15, $1.0 \mathrm{~mL} / \mathrm{min}, \mathrm{t}_{\mathrm{major}}=$ 49.9 min, $\left.\mathrm{t}_{\text {minor }}=52.8 \mathrm{~min}\right) .[\alpha]_{\mathrm{D}}{ }^{20}=-23.5\left(\right.$ c $1.37, \mathrm{CH}_{2} \mathrm{Cl}_{2}, 90 \%$ ee $) .{ }^{1} \mathrm{H}$ NMR (300 MHz, $\left.\mathrm{CDCl}_{3}\right): \delta=7.45-7.41(\mathrm{~m}, 1 \mathrm{H}), 7.33-7.18(\mathrm{~m}, 7 \mathrm{H}), 7.08-7.02(\mathrm{~m}, 1 \mathrm{H})$, 6.82 (s, 1H), 5.15 (t, $J=8.0 \mathrm{~Hz}, 1 \mathrm{H}$ ), 5.00 (dd, $J=12.4,7.6 \mathrm{~Hz}, 1 \mathrm{H}$ ), 4.89 (dd, $J=12.3,8.4$ $\mathrm{Hz}, 1 \mathrm{H}), 3.67$ (s, 3H). ${ }^{13} \mathrm{C}$ NMR (75 MHz, $\left.\mathrm{CDCl}_{3}\right): \delta=139.3,137.1,128.8,127.7,127.4$, 126.4, 126.3, 122.1, 119.4, 118.9, 112.6, 109.5, 79.4, 41.4, 32.7. Lit. $^{2}[\alpha]_{\mathrm{D}}{ }^{20}=+10.8(c 0.6$, $\mathrm{CH}_{2} \mathrm{Cl}_{2}, 31 \%$ ee) for $(S)$ configuration.

3-[1-(2-Methoxyphenyl)-2-nitroethyl]-1H-indole (7e). Prepared according to the general<smiles>COc1ccccc1C(C[N+](=O)[O-])c1c[nH]c2ccccc12</smiles>
procedure using 2-methoxy- $\beta$-nitrostyrene $\mathbf{6 b}(89.6 \mathrm{mg}, 0.50 \mathrm{mmol})$ and indole $5 a$ (57.0 mg, $0.5 \mathrm{mmol})$. Purified by column chromatography (20\% AcOEt in petroleum ether) to yield $145.1 \mathrm{mg}(99 \%)$ of $\mathbf{7 e}$ as an oil. The ee was determined by chiral HPLC on Daicel Chiracel OD-H column (hexane: 2-propanol 70:30, $0.9 \mathrm{~mL} / \mathrm{min}, \mathrm{t}_{\text {major }}=12.7 \mathrm{~min}, \mathrm{t}_{\text {minor }}=14.5$ $\min ) .[\alpha]_{\mathrm{D}}^{20}=-49.9$ (c 1.00, $\mathrm{CH}_{2} \mathrm{Cl}_{2}, 77 \%$ ee). ${ }^{1} \mathrm{H}$ NMR $(300 \mathrm{MHz}$, $\left.\mathrm{CDCl}_{3}\right): \delta=7.96(\mathrm{~s}, 1 \mathrm{H}), 7.45$ (d, $\left.J=7.8 \mathrm{~Hz}, 1 \mathrm{H}\right) 7.26-6.99(\mathrm{~m}, 6 \mathrm{H}), 6.86$ (d, $J=7.8 \mathrm{~Hz}$, $1 \mathrm{H}), 6.79$ (t, $J=7.2 \mathrm{~Hz}, 1 \mathrm{H}$ ), 5.58 (dd, $J=8.6,7.0 \mathrm{~Hz}, 1 \mathrm{H}), 5.03-4.89$ (m, 2H), 3.85 (s, 3H). ${ }^{13} \mathrm{C}$ NMR $\left(75 \mathrm{MHz}, \mathrm{CDCl}_{3}\right): \delta=156.8,136.3,128.8,128.6,127.1,126.4,122.3,121.9$, $120.7,119.6,119.0,113.7,111.2,110.7,78.0,55.4,35.4$. Lit. $^{2}[\alpha]_{\mathrm{D}}{ }^{20}=+49.6\left(c 0.75, \mathrm{CH}_{2} \mathrm{Cl}_{2}\right.$, $61 \%$ ee). 
3-[1-(2-Chlorophenyl)-2-nitroethyl]-1H-indole (7f). Prepared according to the general procedure using 2-chloro- $\beta$-nitrostyrene $\mathbf{6 c}(96.8 \mathrm{mg}, 0.50 \mathrm{mmol})$ and indole $5 \mathbf{a}(57.0 \mathrm{mg}$,

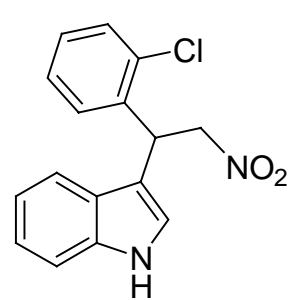

0.5 mmol). Purified by column chromatography (20\% AcOEt in petroleum ether) to yield $153.1 \mathrm{mg}$ (99\%) of $\mathbf{7 f}$ as an oil. The ee was determined by chiral HPLC on Daicel Chiracel OD-H column (hexane: 2-propanol 70:30, $\left.0.9 \mathrm{~mL} / \mathrm{min}, \mathrm{t}_{\text {major }}=16.1 \mathrm{~min}, \mathrm{t}_{\text {minor }}=25.4 \mathrm{~min}\right) \cdot[\alpha]_{\mathrm{D}}{ }^{20}$ $=-52.1$ ( $c$ 1.45, $\mathrm{CH}_{2} \mathrm{Cl}_{2}, 73 \%$ ee). ${ }^{1} \mathrm{H}$ NMR (300 MHz, $\left.\mathrm{CDCl}_{3}\right): \delta=8.08$ (s, 1H), $7.42(\mathrm{~m}, 2 \mathrm{H}), 7.33(\mathrm{~d}, J=8.1 \mathrm{~Hz}, 1 \mathrm{H}) 7.24-7.04(\mathrm{~m}, 6 \mathrm{H}), 5.73(\mathrm{t}$, $J=8.0 \mathrm{~Hz}, 1 \mathrm{H}), 5.03-4.91(\mathrm{~m}, 2 \mathrm{H}) .{ }^{13} \mathrm{C}$ NMR $\left(75 \mathrm{MHz}, \mathrm{CDCl}_{3}\right): \delta=136.4,133.8,130.1$, 128.9, 128.8, 127.2, 126.1, 122.7, 121.9, 120.5, 119.9, 118.8, 113.1, 111.4, 77.6, 37.9. . it. ${ }^{2}$ $[\alpha]_{\mathrm{D}}{ }^{20}=+79.4\left(c 0.95, \mathrm{CH}_{2} \mathrm{Cl}_{2}, 72 \%\right.$ ee $)$.

3-[1-Naphth-2-yl-2-nitroethyl]-1H-indole (7g). Prepared according to the general

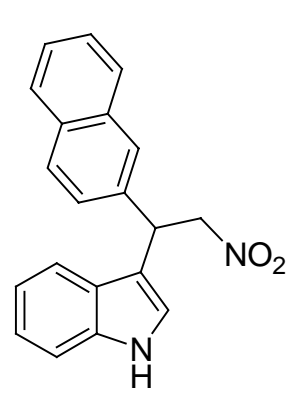
procedure using 2-(2-nitrovinyl)naphthalene 6d (99.6 $\mathrm{mg}, 0.50 \mathrm{mmol})$ and indole $5 a$ ( $57.0 \mathrm{mg}, 0.5 \mathrm{mmol})$. Purified by column chromatography (20\% AcOEt in petroleum ether) to yield $156.0 \mathrm{mg}$ (99\%) of $\mathbf{7 g}$ as an oil. The ee was determined by chiral HPLC on Daicel Chiracel OD-H column (hexane: 2-propanol 70:30, $0.9 \mathrm{~mL} / \mathrm{min}, \mathrm{t}_{\text {major }}=23.6 \mathrm{~min}, \mathrm{t}_{\text {minor }}=28.8$ $\min ) .[\alpha]_{\mathrm{D}}{ }^{20}=-15.6$ (c 1.32, $\mathrm{CH}_{2} \mathrm{Cl}_{2}, 89 \%$ ee). ${ }^{1} \mathrm{H} \mathrm{NMR}(300 \mathrm{MHz}$, $\left.\mathrm{CDCl}_{3}\right): \delta=8.24(\mathrm{~d}, J=7.8 \mathrm{~Hz}, 1 \mathrm{H}), 7.90(\mathrm{~s}, 1 \mathrm{H}), 7.85(\mathrm{~m}, 1 \mathrm{H}), 7.74(\mathrm{t}, J$ $=4.6 \mathrm{~Hz}, 1 \mathrm{H}), 7.52-7.11(\mathrm{~m}, 7 \mathrm{H}), 7.01(\mathrm{t}, J=7.4 \mathrm{~Hz}, 1 \mathrm{H}), 6.84(\mathrm{~s}, 1 \mathrm{H})$, $6.03(\mathrm{t}, J=7.8 \mathrm{~Hz}, 1 \mathrm{H}), 5.05-4.98(\mathrm{~m}, 2 \mathrm{H}) .{ }^{13} \mathrm{C} \mathrm{NMR}\left(75 \mathrm{MHz}, \mathrm{CDCl}_{3}\right): \delta=136.4,134.5$, 134.1, 131.0, 129.1, 128.2, 126.8, 126.0, 125.9, 125.3, 124.6, 122.60, 122.56, 119.9, 118.7, 114.0, 111.4, 78.4, 36.9. IR (neat): 3433, 3056, 1550, 1458, 1377, 777, $737 \mathrm{~cm}^{-1}$. MS (70 eV, EI): $m / z$ (\%) $316\left(\mathrm{M}^{+}, 60\right), 269$ (100), 256 (70), 241 (14), 153 (78), 127 (23), 115 (20). HRMS (EI) calcd for $\mathrm{C}_{20} \mathrm{H}_{16} \mathrm{~N}_{2} \mathrm{O}_{2}$ : 316.12118, found: 316.12139.

3-[1-Furan-2-yl-2-nitroethyl]-1H-indole (7h). Prepared according to the general procedure<smiles>O=[N+]([O-])CC(c1ccco1)c1c[nH]c2ccccc12</smiles>
using 2-(2-nitrovinyl)furan 6e (69.6 $\mathrm{mg}, 0.50 \mathrm{mmol})$ and indole 5a (57.0 mg, $0.5 \mathrm{mmol})$. Purified by column chromatography (20\% AcOEt in petroleum ether) to yield $107.6 \mathrm{mg}(85 \%)$ of $\mathbf{7 h}$ as an oil. The ee was determined by chiral HPLC on Daicel Chiracel OD-H column (hexane: 2-propanol 70:30, $\left.0.9 \mathrm{~mL} / \mathrm{min}, \mathrm{t}_{\text {major }}=14.6 \mathrm{~min}, \mathrm{t}_{\text {minor }}=20.2 \mathrm{~min}\right) \cdot[\alpha]_{\mathrm{D}}{ }^{20}$ $=+37.9$ (c 1.04, $\mathrm{CH}_{2} \mathrm{Cl}_{2}, 81 \%$ ee). ${ }^{1} \mathrm{H}$ NMR (300 MHz, $\left.\mathrm{CDCl}_{3}\right): \delta=8.03$ (s, 1H), 7.53 (d, $J=7.8 \mathrm{~Hz}, 1 \mathrm{H}), 7.34-7.04$ (m, 4H), 6.99 (d, $J=1.5 \mathrm{~Hz}, 1 \mathrm{H}), 6.27$ (dd, $J=$ 1.8, $3.0 \mathrm{~Hz}, 1 \mathrm{H}$ ), 6.12 (d, $J=3.3 \mathrm{~Hz}, 1 \mathrm{H}), 5.21$ (t, $J=7.6 \mathrm{~Hz}, 1 \mathrm{H}), 5.01$ (dd, $J=12.4,8.2 \mathrm{~Hz}$, $1 \mathrm{H}), 4.86$ (dd, $J=12.6,7.5 \mathrm{~Hz}, 1 \mathrm{H}) .{ }^{13} \mathrm{C}$ NMR $\left(75 \mathrm{MHz}, \mathrm{CDCl}_{3}\right.$ ): $\delta=152.1,142.2,136.2$, $125.5,122.7,122.5,119.9,118.6,111.5,111.3,110.4,107.3,77.8,35.6$. $^{2}{ }^{2}[\alpha]_{\mathrm{D}}{ }^{20}=-78(c$ $1.0, \mathrm{CH}_{2} \mathrm{Cl}_{2}, 78 \%$ ee)

3-[1-Nitromethylbutyl]-1H-indole (7i). Prepared according to the general procedure using 


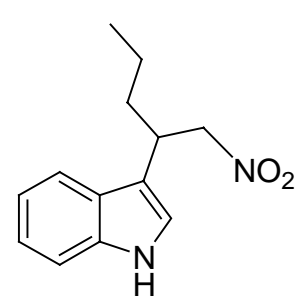

1-nitropent-1-ene $\mathbf{6 f}$ (57.6 mg, $0.50 \mathrm{mmol})$ and indole $5 \mathbf{a}$ (57.0 mg, 0.5 mmol). Purified by column chromatography (20\% AcOEt in petroleum ether) to yield $106.4 \mathrm{mg}$ (91\%) of $\mathbf{7 i}$ as an oil. The ee was determined by chiral HPLC on Daicel Chiracel OD-H column (hexane: 2-propanol $\left.90: 10,1.0 \mathrm{~mL} / \mathrm{min}, \mathrm{t}_{\text {major }}=31.8 \mathrm{~min}, \mathrm{t}_{\text {minor }}=34.8 \mathrm{~min}\right) .[\alpha]_{\mathrm{D}}{ }^{20}=+39.6(c$ 1.12, $\mathrm{CH}_{2} \mathrm{Cl}_{2}, 92 \%$ ee). ${ }^{1} \mathrm{H}$ NMR (300 $\left.\mathrm{MHz}, \mathrm{CDCl}_{3}\right): \delta=7.99$ (s, $\left.1 \mathrm{H}\right)$, 7.60 (d, $J=7.8 \mathrm{~Hz}, 1 \mathrm{H}), 7.27(\mathrm{~d}, J=8.1 \mathrm{~Hz}, 1 \mathrm{H}), 7.20-7.08(\mathrm{~m}, 2 \mathrm{H}), 6.88$ (d, $J=1.0 \mathrm{~Hz}$, 1H), 4.65-4.53 (m, 2H), 3.79-3.74 (m, 1H), 1.84-1.68 (m, 2H), 1.32-1.22 (m, 2H), 0.84 (t, $J$ $=7.4 \mathrm{~Hz}, 1 \mathrm{H}) .{ }^{13} \mathrm{C}$ NMR $\left(75 \mathrm{MHz}, \mathrm{CDCl}_{3}\right): \delta=136.3,126.0,122.2,121.9,119.5,118.5$, 113.7, 111.5, 80.5, 36.0, 34.5, 20.2, 13.7. Lit. $^{2}[\alpha]_{\mathrm{D}}{ }^{20}=-51.1$ (c 0.45, EtOH, 70\% ee).

3-[1-Nitromethyldecyl]-1H-indole (7j). Prepared according to the general procedure using<smiles>CCC(C[N+](=O)[O-])c1c[nH]c2ccccc12</smiles>
1-nitroundec-1-ene $\mathbf{6 g}$ (99.6 mg, $0.50 \mathrm{mmol})$ and indole $5 \mathbf{5}(57.0 \mathrm{mg}$, $0.5 \mathrm{mmol}$ ). Purified by column chromatography (20\% AcOEt in petroleum ether) to yield $134.2 \mathrm{mg}(91 \%)$ of $\mathbf{7} \mathbf{j}$ as an oil. The ee was determined by chiral HPLC on Daicel Chiracel OD-H column (hexane: 2-propanol 90:10, $1.0 \mathrm{~mL} / \mathrm{min}, \mathrm{t}_{\text {major }}=23.0 \mathrm{~min}, \mathrm{t}_{\text {minor }}=26.4 \mathrm{~min}$ ). $[\alpha]_{\mathrm{D}}{ }^{20}=+22.9\left(\right.$ c $1.29, \mathrm{CH}_{2} \mathrm{Cl}_{2}, 89 \%$ ee). ${ }^{1} \mathrm{H}$ NMR (300 MHz, $\left.\mathrm{CDCl}_{3}\right)$ : $\delta=8.05$ (s, 1H), 7.62 (d, $J=8.1 \mathrm{~Hz}, 1 \mathrm{H}), 7.34$ (d, $J=7.8 \mathrm{~Hz}, 1 \mathrm{H}), 7.23-7.10(\mathrm{~m}, 2 \mathrm{H}), 6.98$ (d, $J=2.4 \mathrm{~Hz}, 1 \mathrm{H}), 4.70-4.58$ (m, 2H), 3.82-3.73 (m, 1H), 1.90-1.72 (m, 2H), 1.27-1.20 (m, $14 \mathrm{H}), 0.86$ (t, $J=6.8 \mathrm{~Hz}, 1 \mathrm{H}) .{ }^{13} \mathrm{C}$ NMR $\left(75 \mathrm{MHz}, \mathrm{CDCl}_{3}\right): \delta=136.4,126.1,122.3,121.9$, 119.7, 118.7, 114.0, 111.5, 80.5, 36.3, 32.4, 31.8, 29.5, 29.4, 29.2, 27.1, 22.6, 14.1. IR (neat): 3422, 2925, 2854, 1548, 1458, 1425, 1380, $741 \mathrm{~cm}^{-1}$. MS (70 eV, EI): $m / z(\%) 316\left(\mathrm{M}^{+}, 46\right)$, 270 (21), 256 (16), 157 (46), 143 (80), 130 (100), 115 (10). HRMS (EI) calcd for $\mathrm{C}_{19} \mathrm{H}_{28} \mathrm{~N}_{2} \mathrm{O}_{2}$ : 316.21508, found: 316.21541.

3-[1-Nitromethyl-3-phenylpropyl]-1H-indole (7k). Prepared according to the general

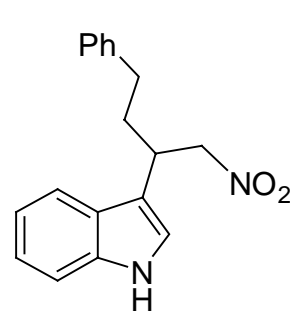
procedure using 1-nitro-4-phenylbut-1-ene $\mathbf{6 h}(88.6 \mathrm{mg}, 0.50 \mathrm{mmol})$ and indole 5 a $(57.0 \mathrm{mg}, 0.5 \mathrm{mmol})$. Purified by column chromatography (20\% AcOEt in petroleum ether) to yield $140.0 \mathrm{mg}$ (88\%) of $\mathbf{7 k}$ as an oil. The ee was determined by chiral HPLC on Daicel Chiracel OD-H column (hexane: 2-propanol 85:15, $1.0 \mathrm{~mL} / \mathrm{min}, \mathrm{t}_{\text {major }}=32.5 \mathrm{~min}, \mathrm{t}_{\text {minor }}=38.6$ $\min ) .[\alpha]_{\mathrm{D}}{ }^{20}=+19.8\left(\right.$ c $1.61, \mathrm{CH}_{2} \mathrm{Cl}_{2}, 93 \%$ ee). ${ }^{1} \mathrm{H}$ NMR $(300 \mathrm{MHz}$, $\left.\mathrm{CDCl}_{3}\right): \delta=8.05$ (s, $\left.1 \mathrm{H}\right), 7.59$ (d, $\left.J=7.8 \mathrm{~Hz}, 1 \mathrm{H}\right), 7.35$ (d, $J=7.8 \mathrm{~Hz}$, 1H), 7.29-7.11 (m, 5H), 7.07 (d, $J=6.9 \mathrm{~Hz}, 2 \mathrm{H}), 7.00$ (d, $J=2.1 \mathrm{~Hz}, 1 \mathrm{H}), 4.69-4.57$ (m, 2H), 3.84-3.74 (m, 1H), 2.67-2.48 (m, 2H), 2.24-2.06 (m, 2H). ${ }^{13} \mathrm{C}$ NMR (75 MHz, $\mathrm{CDCl}_{3}$ ): $\delta=141.2,136.5,128.4,128.3,126.00,125.96,122.4,122.2,119.8,118.7,113.3,111.6,80.4$, 35.9, 33.9, 33.2. IR (neat): 3422, 2920, 1546, 1457, 1381, 743, $701 \mathrm{~cm}^{-1}$. MS (70 eV, EI): $\mathrm{m} / \mathrm{z}$ (\%) 294 (M+, 90), 247 (30), 232 (13), 156 (14), 143 (100), 130 (70), 117 (50), 91 (87). HRMS (EI) calcd for $\mathrm{C}_{18} \mathrm{H}_{18} \mathrm{~N}_{2} \mathrm{O}_{2}$ : 294.13683, found: 294.13690 . 
3-(1-Cyclohexyl-2-nitroethyl)-1H-indole (7l). Prepared according to the general procedure using 2-nitrovinylcyclohexane $\mathbf{6 i}$ (77.6 $\mathrm{mg}, 0.50 \mathrm{mmol})$ and indole 5 a $(57.0 \mathrm{mg}, 0.5 \mathrm{mmol})$. Purified by column chromatography (20\% AcOEt in petroleum ether) to yield $125.2 \mathrm{mg}$

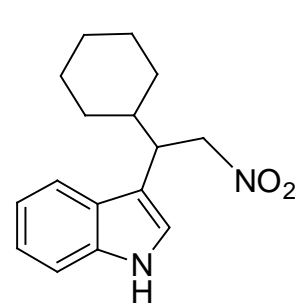
(92\%) of $7 \mathbf{l}$ as an oil. The ee was determined by chiral HPLC on Daicel Chiracel AD column (hexane: 2-propanol 90:10, $1.0 \mathrm{~mL} / \mathrm{min}, \mathrm{t}_{\text {major }}=13.7$ min, $\left.\mathrm{t}_{\text {minor }}=17.4 \mathrm{~min}\right) .[\alpha]_{\mathrm{D}}{ }^{20}=+40.4\left(c 1.36, \mathrm{CH}_{2} \mathrm{Cl}_{2}, 84 \%\right.$ ee). ${ }^{1} \mathrm{H} \mathrm{NMR}$ (300 MHz, $\left.\mathrm{CDCl}_{3}\right): \delta=8.02(\mathrm{~s}, 1 \mathrm{H}), 7.60-7.57(\mathrm{~m}, 1 \mathrm{H}), 7.31-7.28(\mathrm{~m}$, 1H), 7.21-7.08 (m, 2H), 6.89 (d, $J=2.4 \mathrm{~Hz}, 1 \mathrm{H}), 4.79$ (dd, $J=12.0,6.3$ $\mathrm{Hz}, 1 \mathrm{H}$ ), 4.69 (dd, $J=11.8,9.4 \mathrm{~Hz}, 1 \mathrm{H}), 3.69-3.61$ (m, 1H), 1.83-1.61 (m, 6H), 1.29-0.88 (m, 5H). ${ }^{13} \mathrm{C}$ NMR (75 MHz, $\left.\mathrm{CDCl}_{3}\right): \delta=136.1,126.7,122.2,122.1$, 119.5, 119.0, 113.0, 111.4, 78.4, 41.8, 40.4, 31.1, 30.3, 26.2, 26.09, 26.07. IR (neat): 3420, 2925, 2852, 1548, 1457, 1383, 1100, $743 \mathrm{~cm}^{-1}$. MS (70 eV, EI): $m / z(\%) 272\left(\mathrm{M}^{+}, 20\right), 225$ (4), 210 (4), 189 (6), 157 (3), 143 (100), 130 (36), 115 (11), 90 (4), 77 (4). HRMS (EI) calcd for $\mathrm{C}_{16} \mathrm{H}_{20} \mathrm{~N}_{2} \mathrm{O}_{2}$ : 272.15248, found: 272.15221 .

3-(1-tert-butyl-2-nitroethyl)-1H-indole (7m). Prepared according to the general procedure $\checkmark \quad$ using 3,3-dimethyl-1-nitrobut-1-ene $6 \mathbf{j}(57.6 \mathrm{mg}, 0.50 \mathrm{mmol})$ and indole<smiles>CC(C)(C)C(C[N+](=O)[O-])c1c[nH]c2ccccc12</smiles>
5a (57.0 mg, $0.5 \mathrm{mmol}$ ) at $30^{\circ} \mathrm{C}$ for $144 \mathrm{~h}$. Purified by column chromatography (20\% AcOEt in petroleum ether) to yield $104.8 \mathrm{mg}$ (85\%) of $\mathbf{7 m}$ as a pale yellow solid. m.p. $125-127^{\circ} \mathrm{C}$. The ee was determined by chiral HPLC on Daicel Chiracel OD-H column (hexane: 2-propanol 90:10, $\left.1.0 \mathrm{~mL} / \mathrm{min}, \mathrm{t}_{\text {major }}=17.5 \mathrm{~min}, \mathrm{t}_{\text {minor }}=28.7 \mathrm{~min}\right) .[\alpha]_{\mathrm{D}}{ }^{20}=-16.1(c \quad 0.56$, $\mathrm{CH}_{2} \mathrm{Cl}_{2}, 87 \%$ ee). ${ }^{1} \mathrm{H}$ NMR (300 MHz, $\left.\mathrm{CDCl}_{3}\right): \delta=8.06$ (s, $\left.1 \mathrm{H}\right), 7.61(\mathrm{~d}, J=7.5 \mathrm{~Hz}, 1 \mathrm{H})$, 7.29 (d, $J=7.5 \mathrm{~Hz}, 1 \mathrm{H}), 7.19-7.09$ (m, 2H), 6.98 (d, $J=2.4 \mathrm{~Hz}, 1 \mathrm{H}), 4.87-4.71(\mathrm{~m}, 2 \mathrm{H})$, 3.77 (dd, $J=11.1,4.8 \mathrm{~Hz}, 1 \mathrm{H}), 0.99$ (s, 9H). ${ }^{13} \mathrm{C} \mathrm{NMR}\left(75 \mathrm{MHz} \mathrm{CDCl}_{3}\right): \delta=135.7,128.2$, 122.1, 121.9, 119.6, 119.3, 112.9, 111.1, 78.0, 45.5, 34.4, 28.0. IR (neat): 3422, 2962, 1549, 1458, 1380, 1340, 1230, 1103, $743 \mathrm{~cm}^{-1}$. MS (70 eV, EI): $\mathrm{m} / z$ (\%) $246\left(\mathrm{M}^{+}, 17\right), 189$ (6), 143 (100), 130 (8), 115 (9), 57 (17). HRMS (EI) calcd for $\mathrm{C}_{14} \mathrm{H}_{18} \mathrm{~N}_{2} \mathrm{O}_{2}$ : 246.13683, found: 246.13663.

\section{Reference:}

(1) (a) Lucet, D.; Sabelle, S.; Kostelitz, O.; Gall, T. L.; Mioskowski, C. Eur. J. Org. Chem. 1999, 2583. (b) Denmark, S. E.; Marcin, L. J. Org. Chem., 1995, 60, 3221. (c) Denmark, S. E.; Marcin, L. J. Org. Chem. 1993, 58, 3580.

(2) Jia, Y.-X.; Zhu, S.-F.; Yang, Y.; Zhou, Q.-L. J. Org. Chem. 2006, 71, 75.

(3) Herrera, R. P.; Sgarzani, V.; Bernardi, L.; Ricci, A. Angew. Chem. Int. Ed. 2005, 44, 6576. 
Copies of HPLC profiles of nitroalkylated indoles

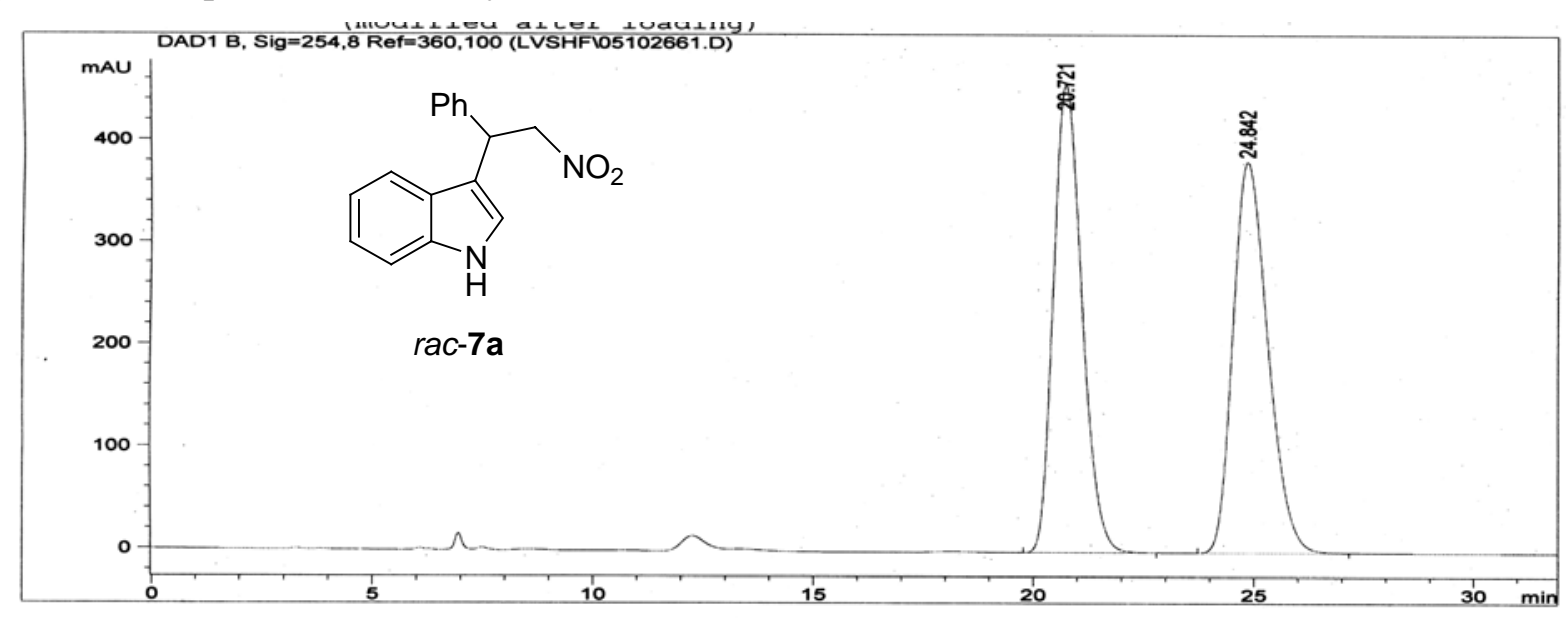

Area Percent Report

$\begin{array}{lll}\text { Sorted By } & : & \text { Signal } \\ \text { Multiplier } & \vdots & 1.0000 \\ \text { Dilution } & : & 1.0000\end{array}$

Signal 1: DAD1 B, Sig=254, 8 Ref=360, 100

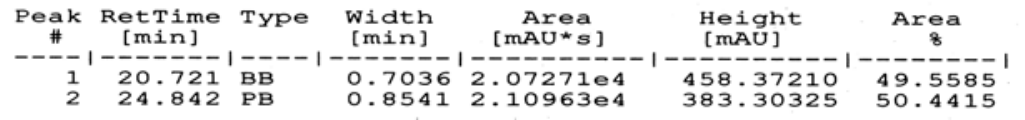

Totals : $4.18234 \mathrm{e}^{4} \quad 841.67535$

Results obtained with enhanced integrator!

$* \star \star$ End of Report $\star \star \star$
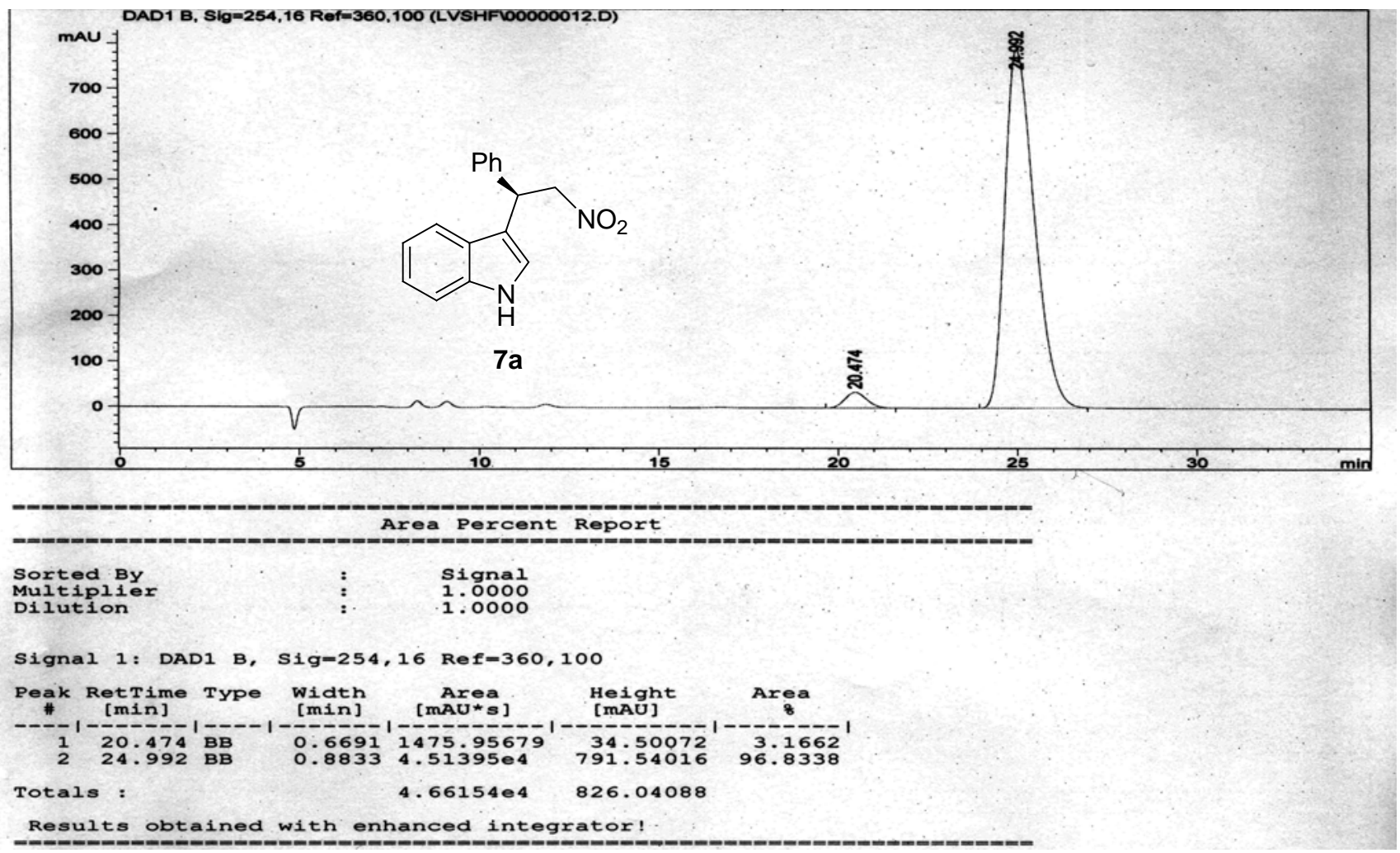

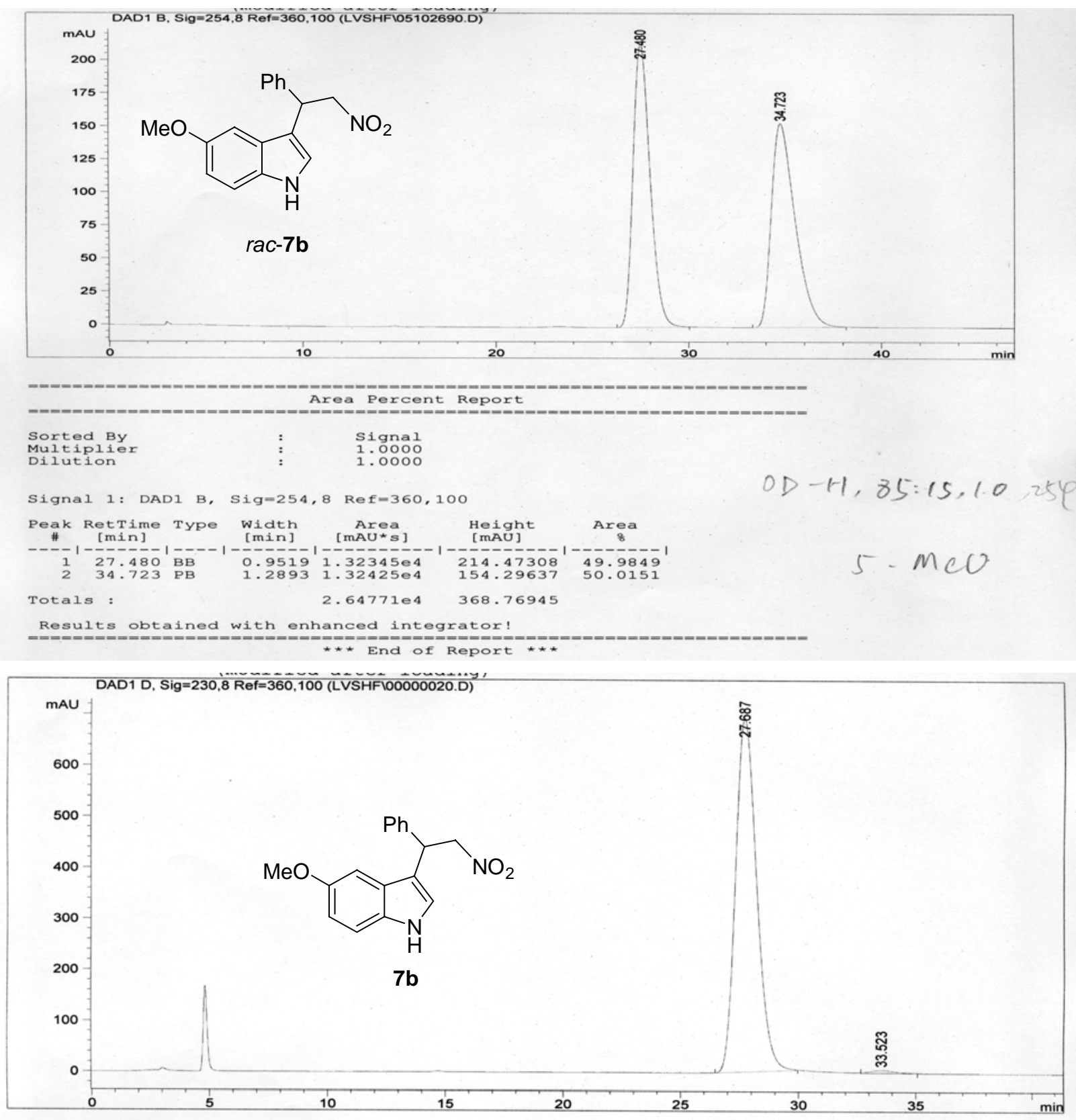

Area Percent Report

$\begin{array}{lll}\text { Sorted By } & : & \text { Signal } \\ \text { Multiplier } & : & 1.0000 \\ \text { Dilution } & : & 1.0000\end{array}$

Signal 1: DAD1 D, $\mathrm{Sig}=230,8 \operatorname{Re} f=360,100$

\begin{tabular}{|c|c|c|c|c|c|c|}
\hline$\underset{\#}{\text { Peak }}$ & $\begin{array}{l}\text { RetTime } \\
\text { [min] }\end{array}$ & туре & $\begin{array}{l}\text { Width } \\
\text { [min] }\end{array}$ & $\begin{array}{c}\text { Area } \\
{\left[\mathrm{mAU}{ }^{\star} \mathrm{s}\right]}\end{array}$ & $\begin{array}{l}\text { Height } \\
\text { [MAU] }\end{array}$ & $\begin{array}{c}\text { Area } \\
8\end{array}$ \\
\hline$\frac{1}{2}$ & $\begin{array}{l}27.687 \\
33.523\end{array}$ & $\begin{array}{l}\text { BB } \\
\text { BP }\end{array}$ & $\begin{array}{l}0.9708 \\
0.8609\end{array}$ & $\begin{array}{c}4.31540 \mathrm{e}^{4} \\
361.44012\end{array}$ & $\begin{array}{r}692.68738 \\
5.40025\end{array}$ & $\begin{array}{r}99.1694 \\
0.8306\end{array}$ \\
\hline Total & : & & & $4.35154 e^{4}$ & 698.08763 & \\
\hline
\end{tabular}

Results obtained with enhanced integrator!

$==1 *==1$
End of Report $\star \star \star$ 


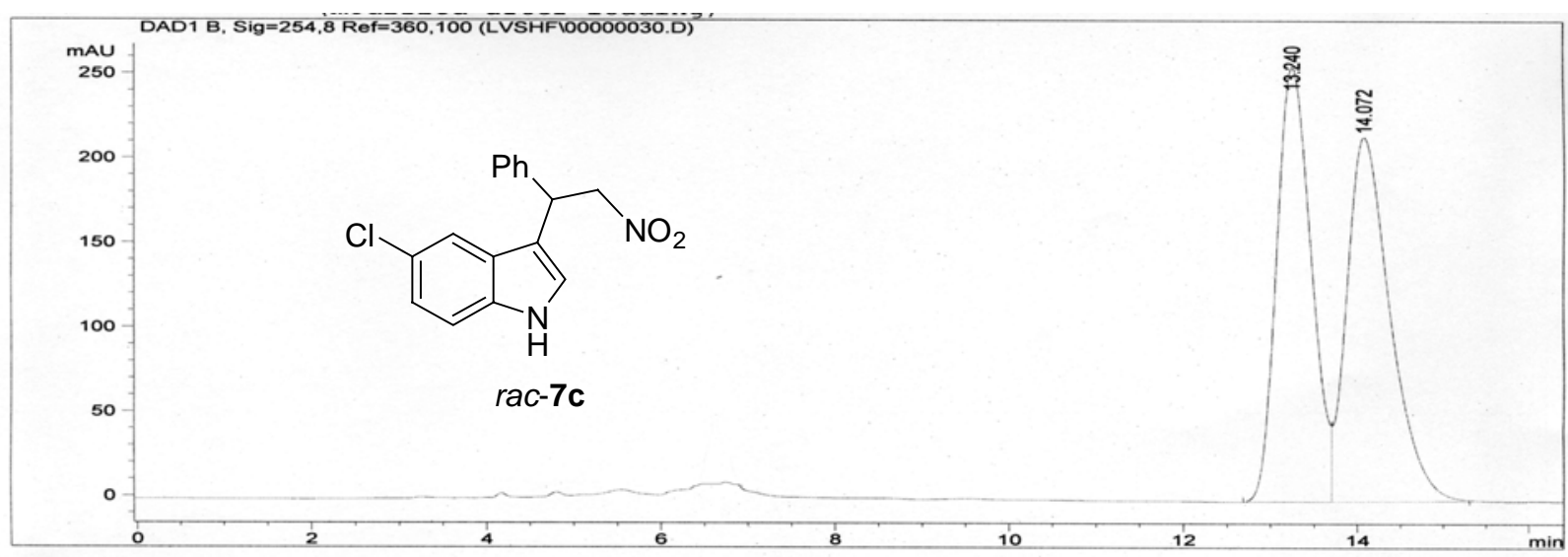

Area Percent Report

$\begin{array}{lll}\text { Sorted By } & : & \text { Signal } \\ \text { Multiplier } & \vdots & 1.0000 \\ \text { Dilution } & \vdots & 1.0000\end{array}$

Signal 1: DAD1 B, Sig-254, 8 Ref=360, 100

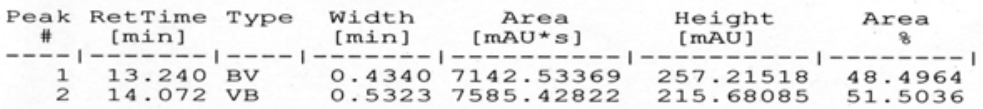

Totals : $\quad 1.4728044 \quad 472.89603$

Results obtained with enhanced integrator!

$\star \star \star$ End of Report $\star \star \star \star$

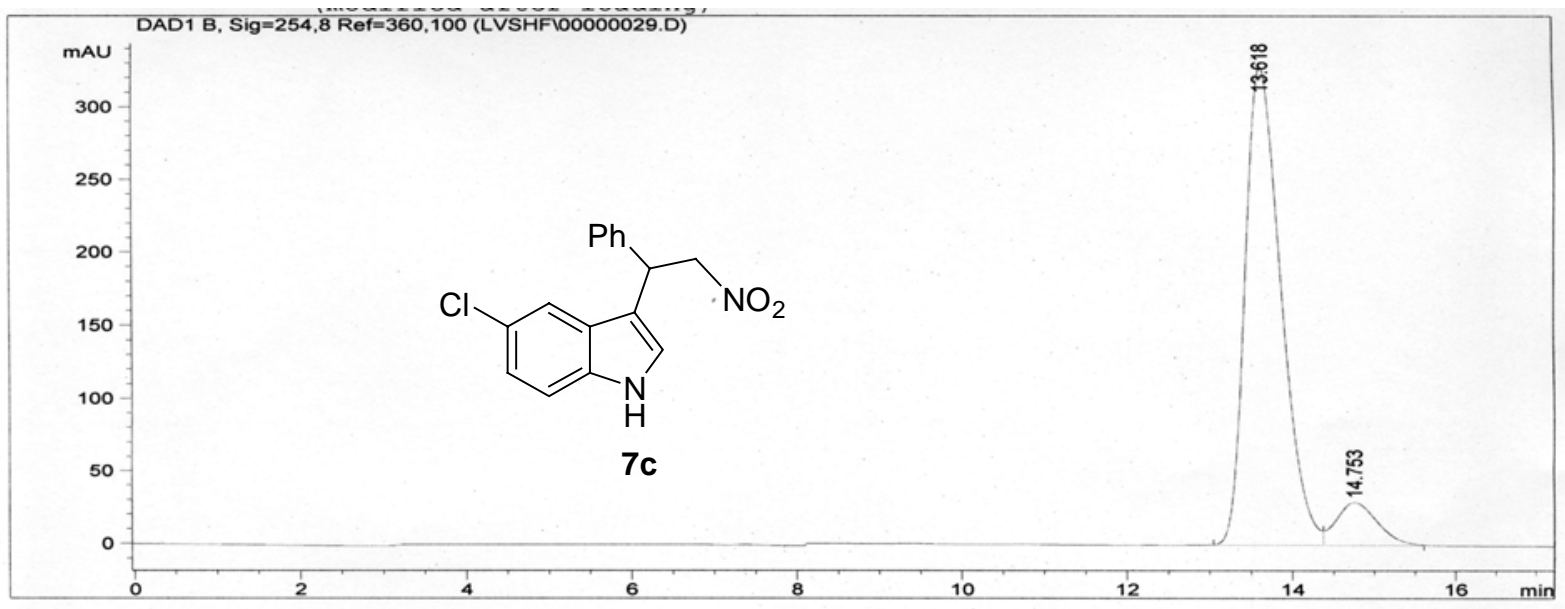

Area Percent Report

$\begin{array}{lll}\text { Sorted By } & : & \text { Signal } \\ \text { Multiplier } & : & 1.0000 \\ \text { Dilution } & : & 1.0000\end{array}$

Multiplier 1.000

Signal 1: DAD1 B, Sig=254, 8 Ref $=360,100$

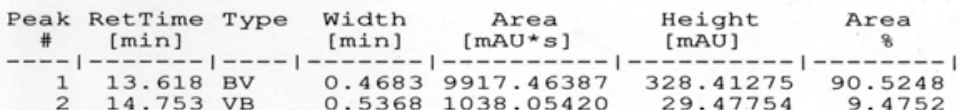

Totals : $\quad 1.09555 e 4 \quad 357.89029$

Results obtained with enhanced integrator!

$\star \star \star$ End of Report *** 


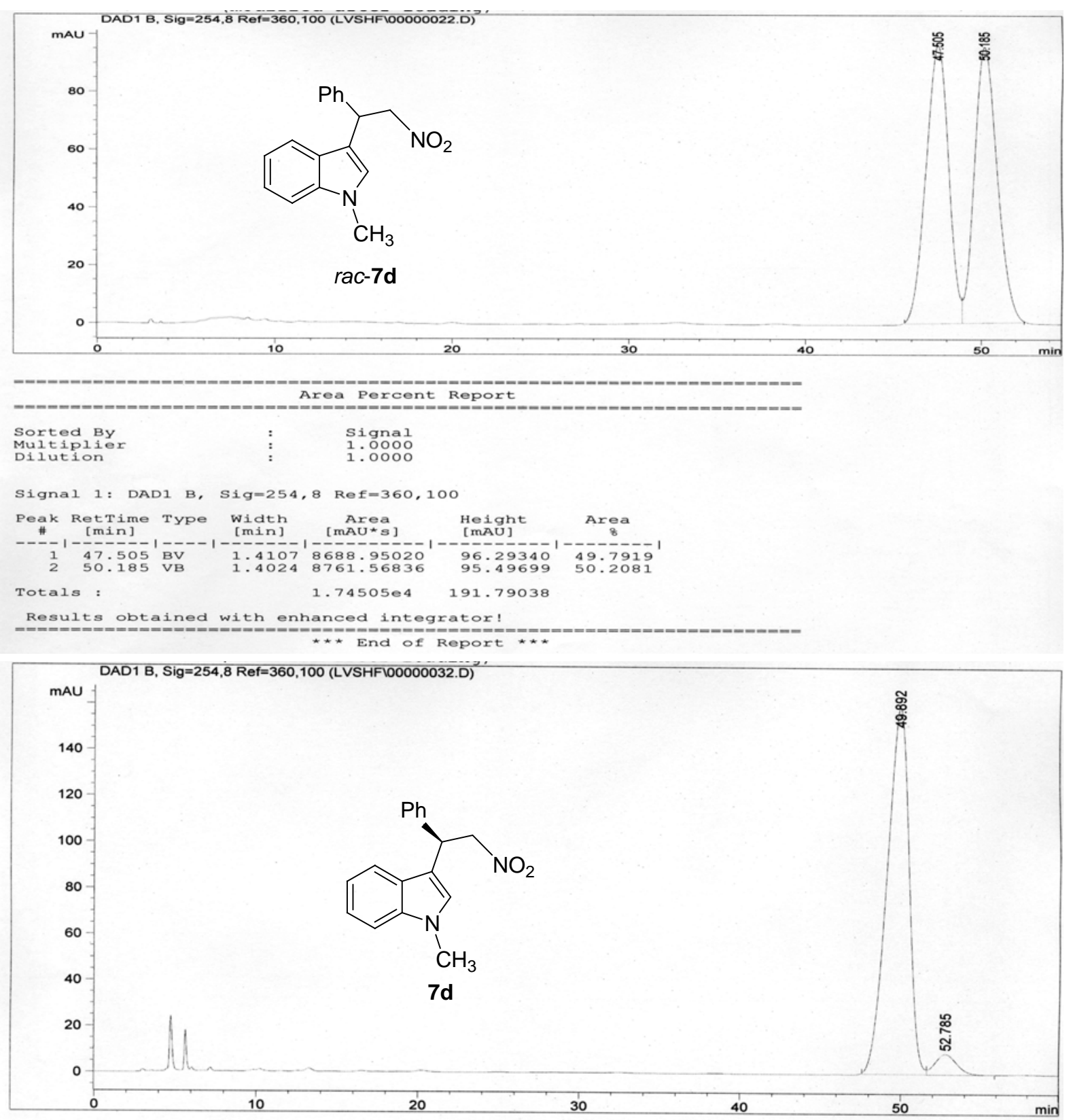

Area Percent Report

Sorted By $\quad: \quad$ Signal

Dilution : : 1.0000

Signal 1: DAD1 B, Sig=254, 8 Ref $=360,100$

\begin{tabular}{|c|c|c|c|c|c|c|}
\hline Peak & $\begin{array}{c}\text { RetTime } \\
\text { [min] }\end{array}$ & Type & $\begin{array}{l}\text { Width } \\
\text { [min] }\end{array}$ & $\begin{array}{c}\text { Area } \\
{\left[\mathrm{mAU}^{\star} \mathrm{s}\right]}\end{array}$ & $\begin{array}{l}\text { Height } \\
\text { [mAU] }\end{array}$ & $\begin{array}{c}\text { Area } \\
8\end{array}$ \\
\hline $\begin{array}{l}1 \\
2\end{array}$ & $\begin{array}{l}49.892 \\
52.785\end{array}$ & $\begin{array}{l}\text { BV } \\
\text { VP }\end{array}$ & $\begin{array}{l}1.4466 \\
1.1899\end{array}$ & $\begin{array}{c}1.51484 \mathrm{e} 4 \\
826.61707\end{array}$ & $\begin{array}{r}159.13583 \\
8.79996\end{array}$ & $\begin{array}{r}94.8256 \\
5.1744\end{array}$ \\
\hline old & & & & $1.59750 e^{4}$ & 167.93579 & \\
\hline
\end{tabular}

Totals : $\quad 1.59750$ e4 167.93579

Results obtained with enhanced integrator!

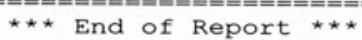



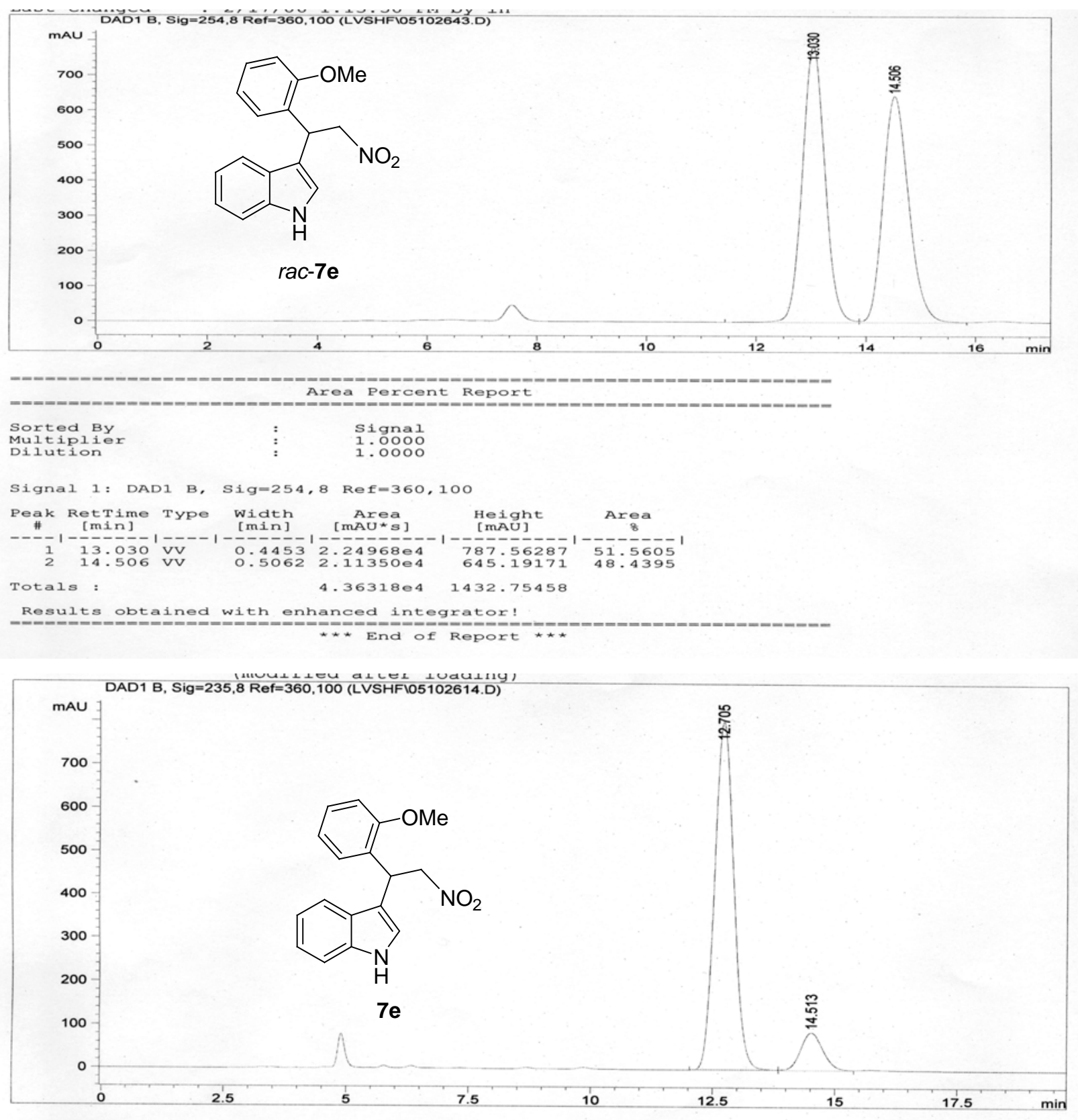

Area Percent Report

$\begin{array}{lll}\text { Sorted By } & : & \text { Signal } \\ \text { Multiplier } & : & 1.0000 \\ \text { Dilution } & : & 1.0000\end{array}$

Signal 1: DAD1 B, Sig=235, 8 Ref=360, 100

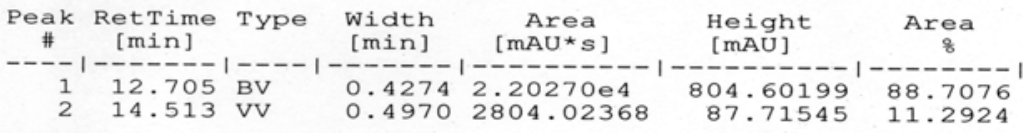

Totals : $\quad 2.48310 e^{4} \quad 892.31744$

Results obtained with enhanced integrator!

$==1 *$ End of Report $\star \star \star$
. 


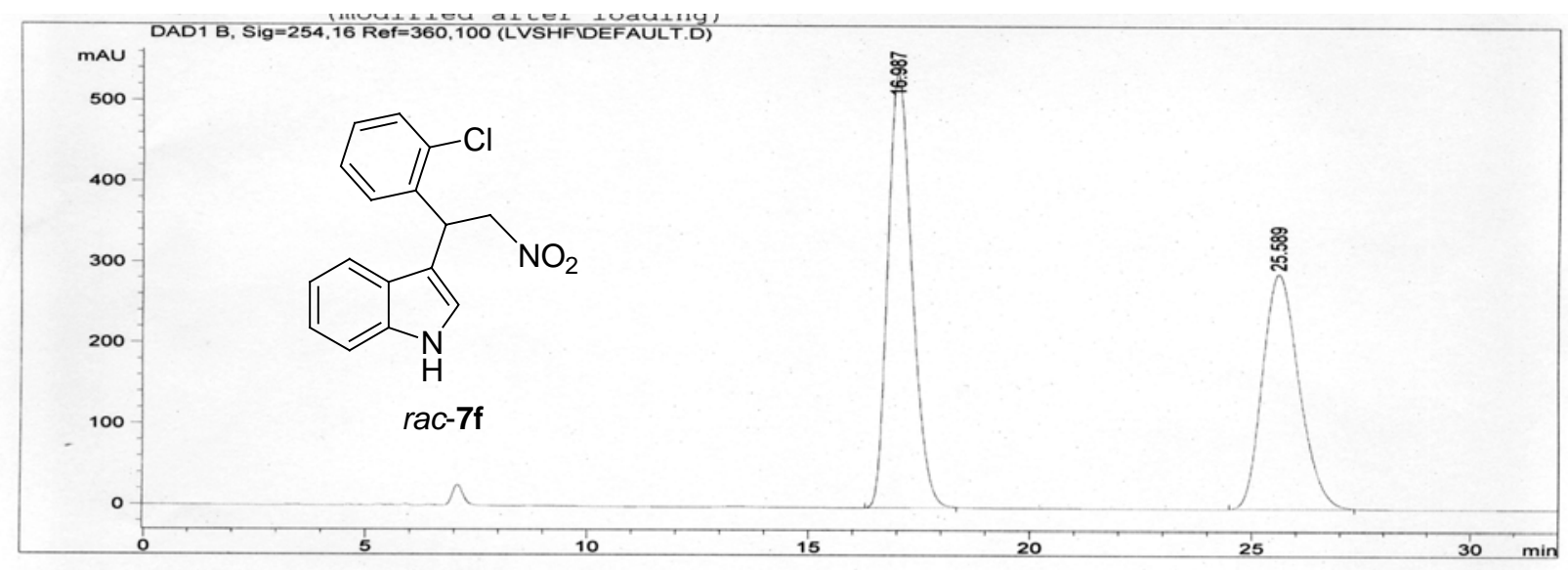

$\begin{array}{lll} & & \text { Area Percent Report } \\ \text { Sorted BY } & & \text { Signal } \\ \text { Multipliex } & \vdots & 1.0000 \\ \text { Dilution } & \vdots & 1.0000\end{array}$

Signal 1: DAD1 B, Sig=254,16 Ref-360,100

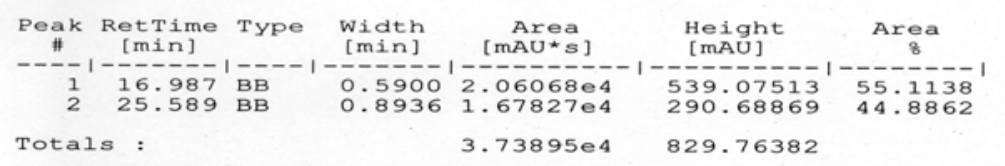

Totals :

$$
\text { 3. } 73895 e 4 \quad 829.76382
$$

Results obtained with enhanced integrator!

$$
\star \star \star \text { End of Report } \star \star \star \star
$$

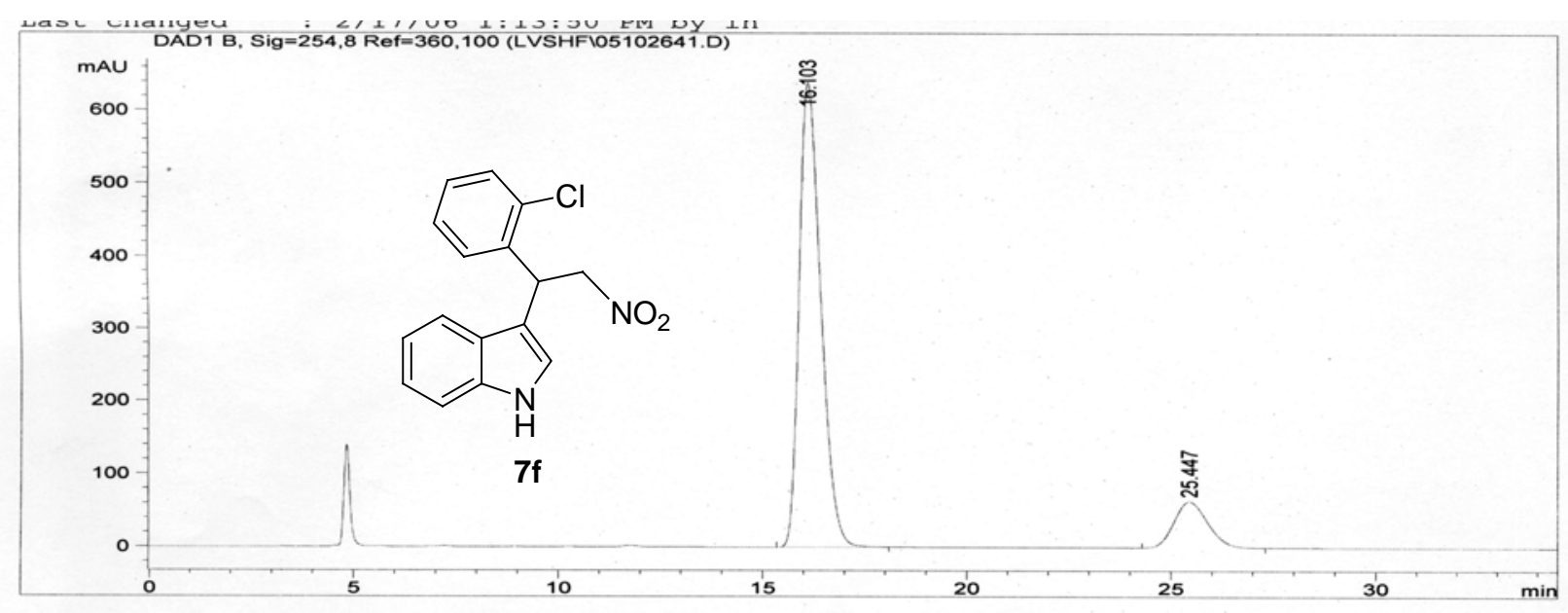

Area Percent Report

$\begin{array}{lll}\text { Sorted By } & : & \text { Signal } \\ \text { Multiplier } & : & 1.0000 \\ \text { Dilution } & : & 1.0000\end{array}$

\begin{tabular}{|c|c|c|c|c|c|c|}
\hline Peak & $\begin{array}{l}\text { Retimime } \\
\text { [min] }\end{array}$ & Type & $\begin{array}{l}\text { Width } \\
{[\text { min] }}\end{array}$ & $\begin{array}{c}\text { Area } \\
{\left[\mathrm{mAU}{ }^{\star} \mathrm{s}\right]}\end{array}$ & $\begin{array}{l}\text { Height } \\
\text { [mAU] }\end{array}$ & $\begin{array}{c}\text { Area } \\
8\end{array}$ \\
\hline$\frac{1}{2}$ & $\begin{array}{l}16.103 \\
16.447 \\
25.44\end{array}$ & $\begin{array}{l}\text { PB } \\
\text { BB }\end{array}$ & $\begin{array}{l}------ \\
0.5700 \\
0.9036\end{array}$ & $\begin{array}{l}2.35452 e^{4} \\
3650.15698\end{array}$ & $\begin{array}{r}638.81201 \\
63.41178\end{array}$ & $\begin{array}{l}-1 .-1--1 \\
86.5780 \\
13.4220\end{array}$ \\
\hline Total & s & & & $2.71953 e^{4}$ & 702.22379 & \\
\hline
\end{tabular}

Signal 1: DAD1 B, Sig=254, 8 Ref $=360,100$

Results obtained with enhanced integrator!

$\star \star \star$ End of Report $\star \star \star *$ 

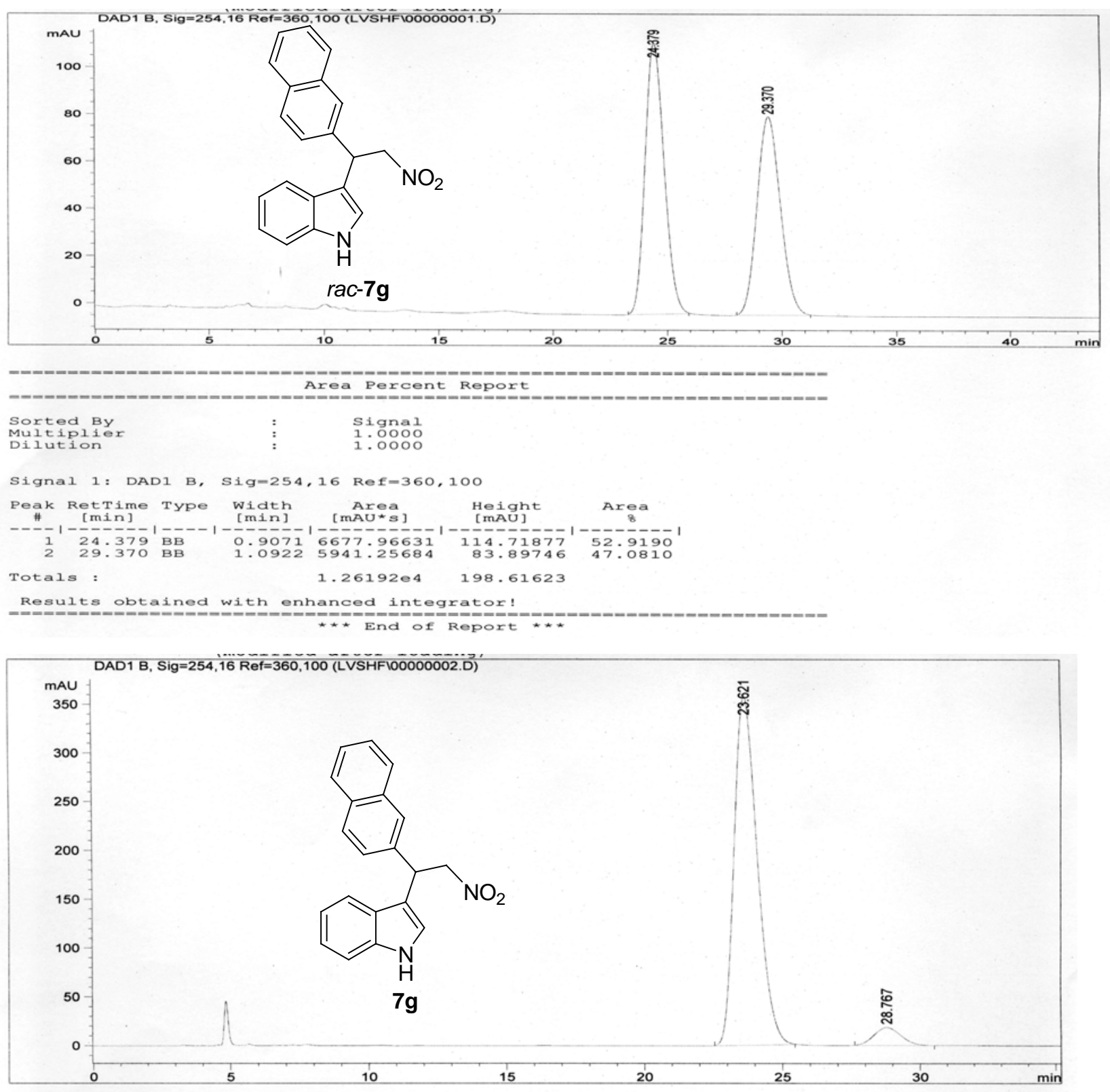

Area Percent Report

$\begin{array}{lll}\text { Sorted By } & : & \text { Signal } \\ \text { Multiplier } & : & 1.0000 \\ \text { Dilution } & : & 1.0000\end{array}$

Signal 1: DAD1 B, Sig=254,16 $\operatorname{Ref}=360,100$

\begin{tabular}{|c|c|c|c|c|c|c|}
\hline$\underset{\#}{\text { Peak }}$ & $\begin{array}{c}\text { RetTime } \\
{[\text { min] }}\end{array}$ & Type & $\begin{array}{l}\text { Width } \\
\text { [min] }\end{array}$ & $\begin{array}{c}\text { Area } \\
{\left[\mathrm{mAU}{ }^{\star} \mathrm{S}\right]}\end{array}$ & $\begin{array}{l}\text { Height } \\
\text { [mAU] }\end{array}$ & $\begin{array}{c}\text { Area } \\
\frac{8}{8}\end{array}$ \\
\hline $\begin{array}{l}1 \\
2\end{array}$ & $\begin{array}{l}23.621 \\
28.767\end{array}$ & $\begin{array}{l}\mathrm{BB} \\
\mathrm{BB}\end{array}$ & $\begin{array}{l}0.9018 \\
1.0351\end{array}$ & $\begin{array}{l}2.08501 \text { e4 } \\
1246.59644\end{array}$ & $\begin{array}{r}357.84937 \\
18.20060\end{array}$ & $\begin{array}{r}94.3585 \\
5.6415\end{array}$ \\
\hline & & & & $2.20967 e 4$ & 376.04997 & \\
\hline
\end{tabular}

Results obtained with enhanced integrator!

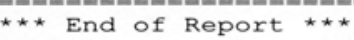



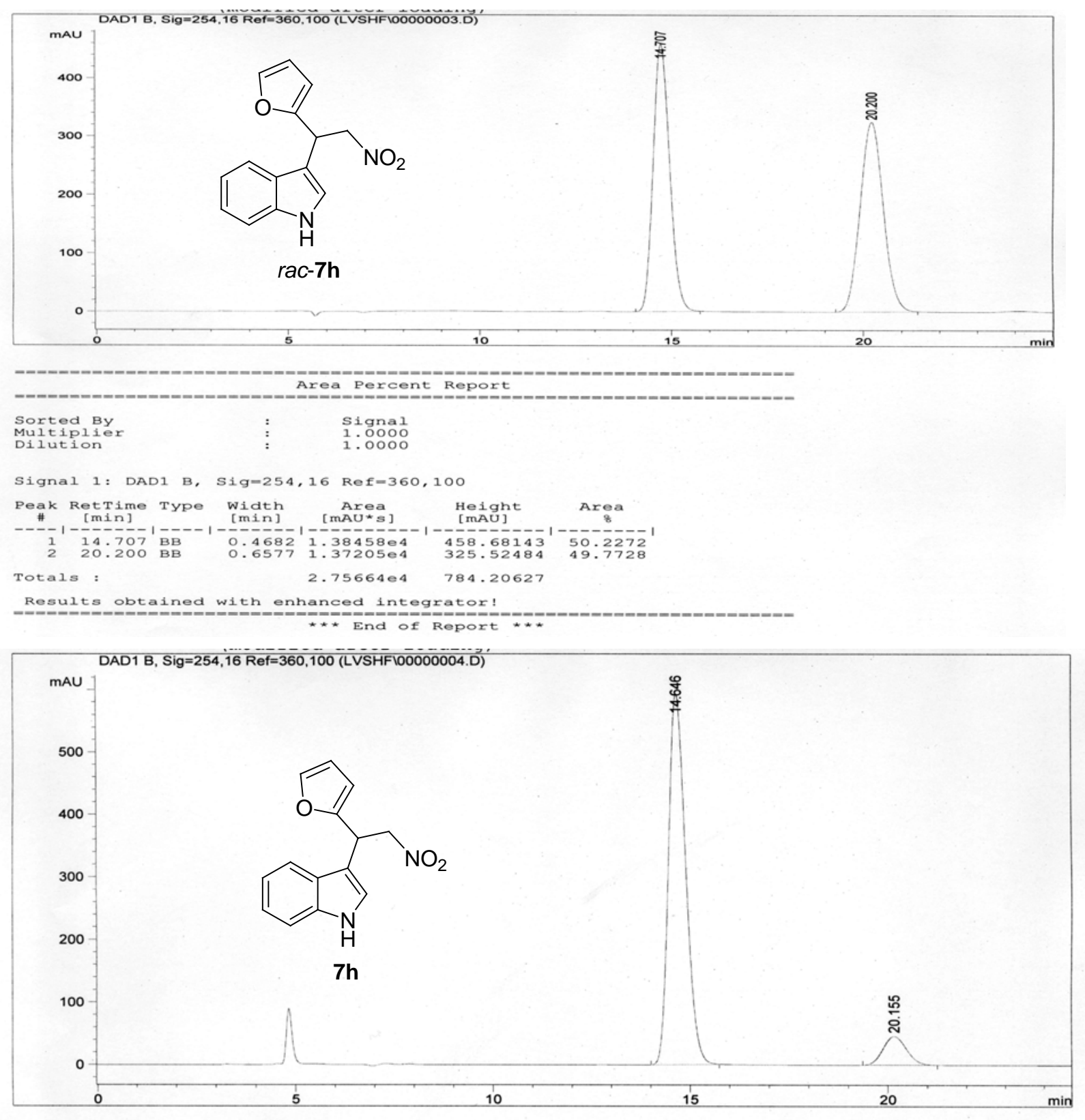

Area Percent Report

Sorted By

Multiplie

Dilution

Signal

1.0000

Signal 1: DAD1 B, Sig=254,16 Ref=360,100

\begin{tabular}{|c|c|c|c|c|c|c|}
\hline $\begin{array}{c}\text { Peak } \\
\#\end{array}$ & $\begin{array}{c}\text { RetTime } \\
\text { [min] }\end{array}$ & Type & $\begin{array}{l}\text { Width } \\
\text { [min] }\end{array}$ & $\begin{array}{c}\text { Area } \\
{\left[\mathrm{mAU}{ }^{\star} \mathrm{s}\right]}\end{array}$ & $\begin{array}{l}\text { Height } \\
\text { [mAU] }\end{array}$ & $\begin{array}{c}\text { Area } \\
8\end{array}$ \\
\hline $\begin{array}{l}1 \\
2\end{array}$ & $\begin{array}{l}14.646 \\
20.155\end{array}$ & $\begin{array}{l}\text { BB } \\
\text { BB }\end{array}$ & $\begin{array}{l}0.4710 \\
0.6466\end{array}$ & $\begin{array}{l}1.79608 \mathrm{e} 4 \\
1913.54919\end{array}$ & $\begin{array}{r}593.68585 \\
46.06454\end{array}$ & $\begin{array}{r}90.3718 \\
9.6282\end{array}$ \\
\hline & & & & $1.98743 \mathrm{e} 4$ & 639.75039 & \\
\hline
\end{tabular}

Results obtained with enhanced integrator!

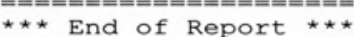




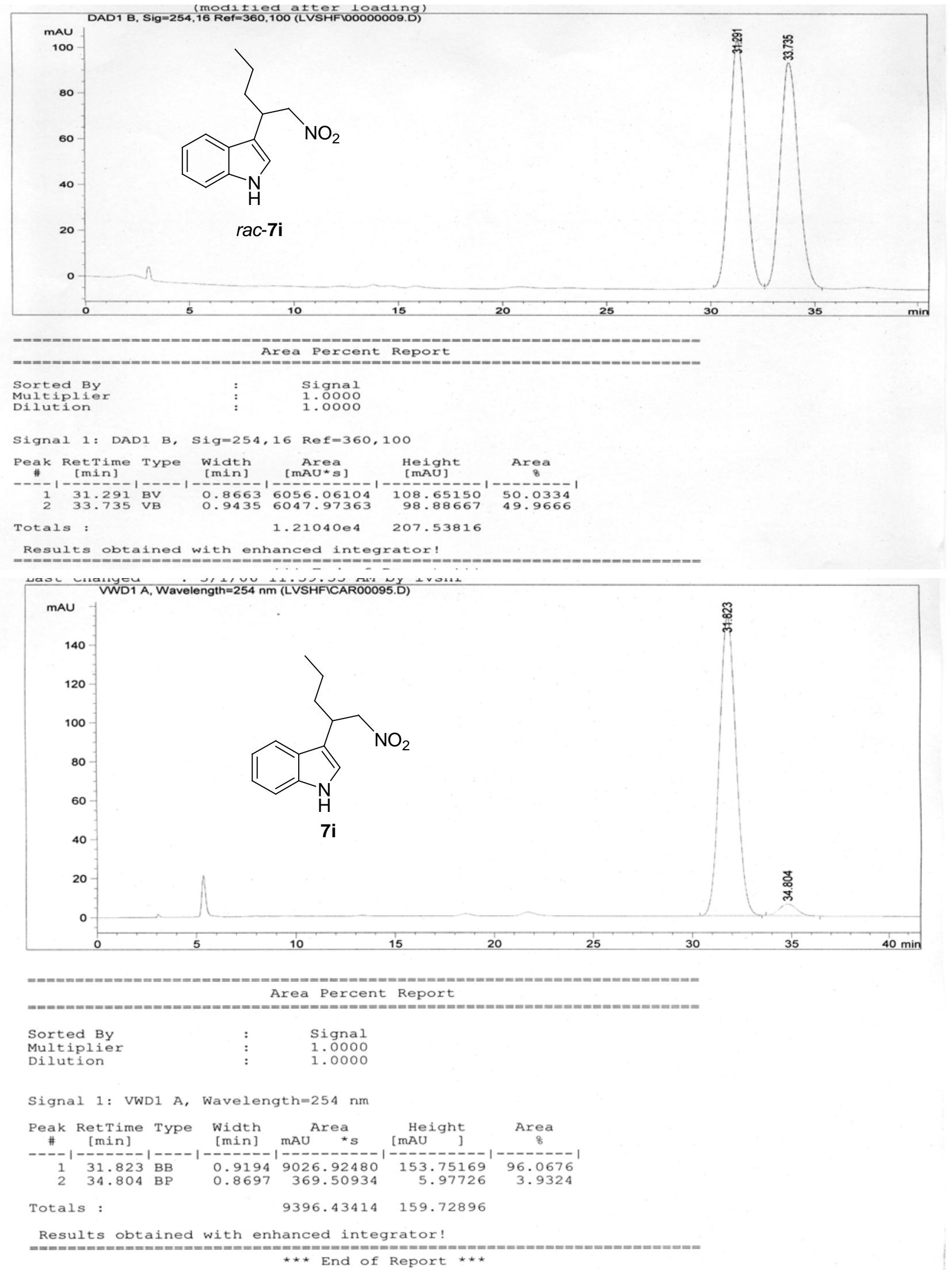



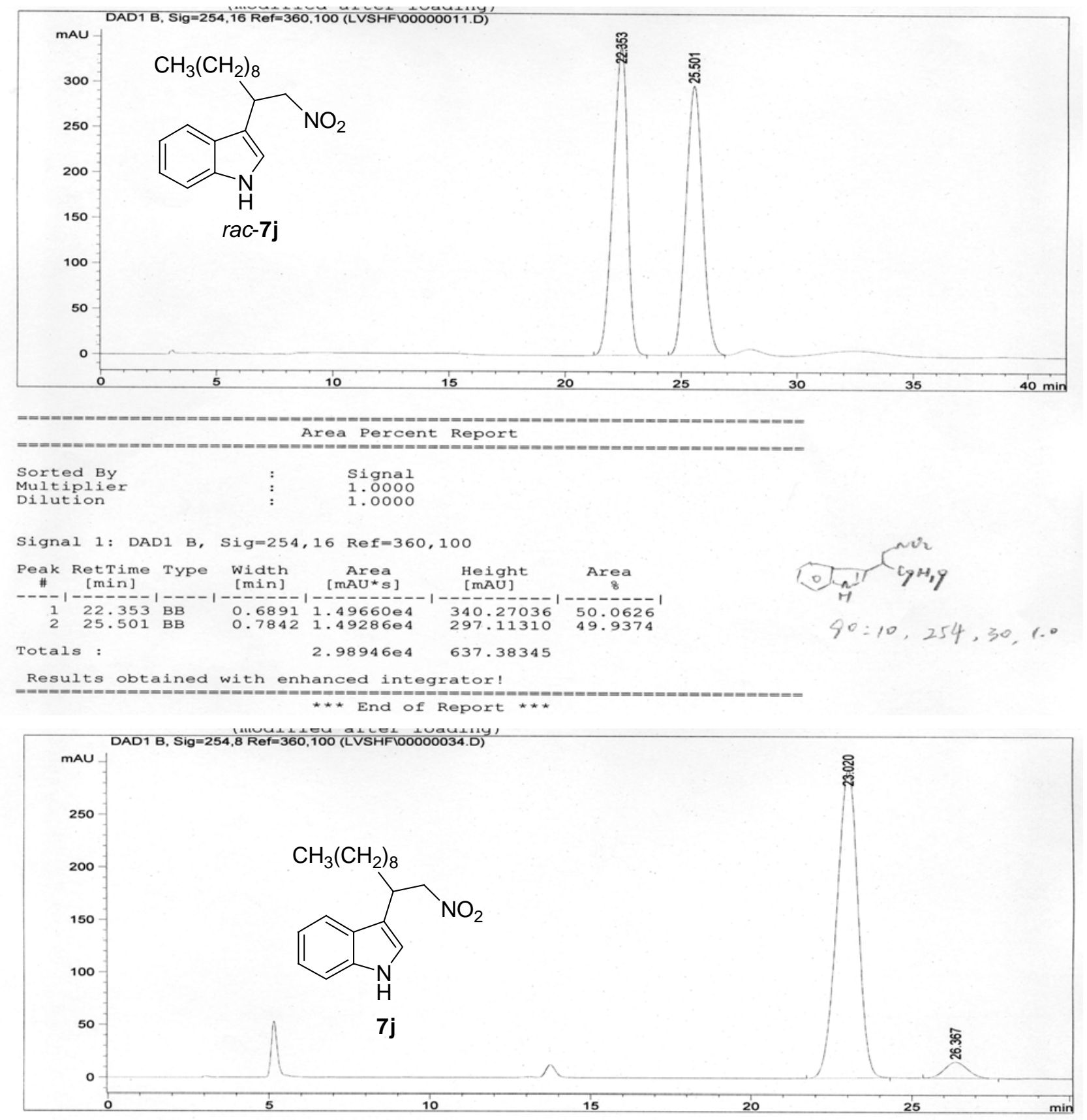

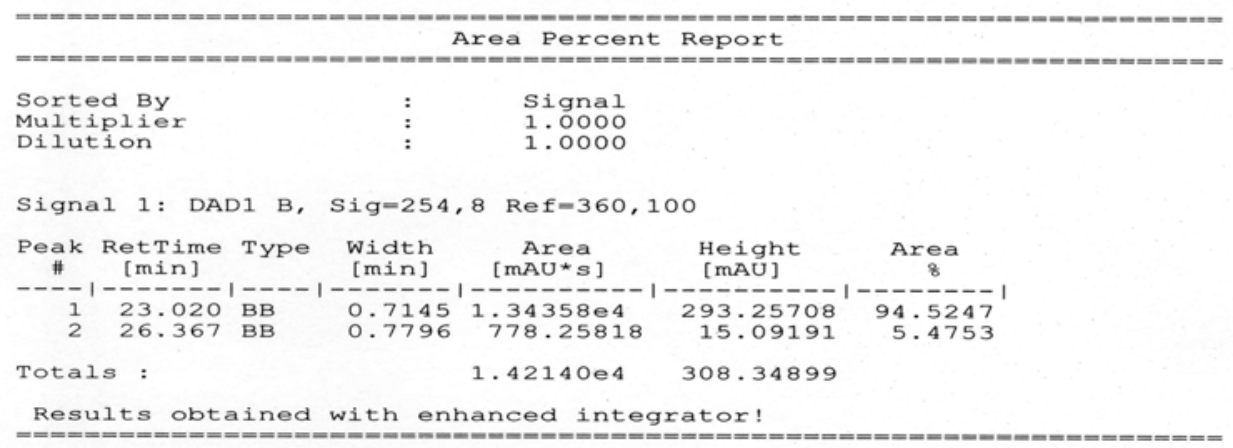

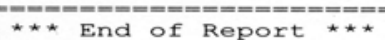




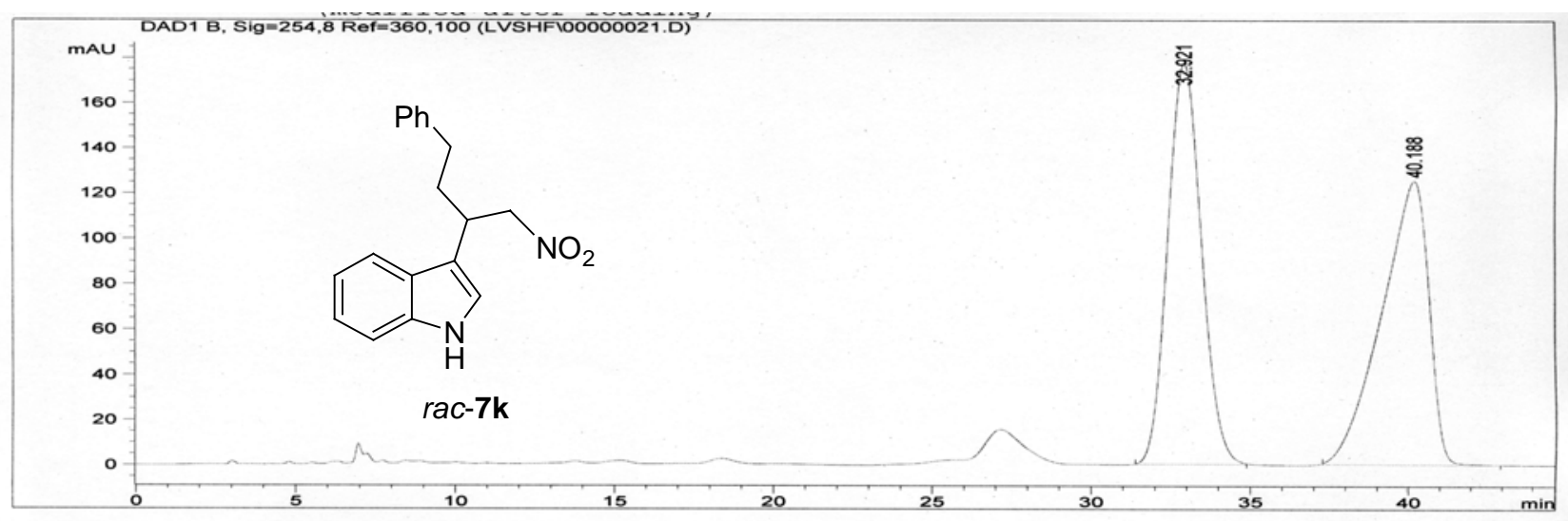

Area percent Report

$\begin{array}{lll}\text { Sorted By } & : & \text { Signal } \\ \text { Multiplier } & \vdots & 1.0000 \\ \text { Dilution } & : & 1.0000\end{array}$

Signal 1: DAD1 B, Sig=254, 8 Ref=360, 100

\begin{tabular}{|c|c|c|c|c|c|c|}
\hline $\begin{array}{c}\text { Peak } \\
-\#\end{array}$ & $\begin{array}{l}\text { Retimime } \\
\text { [min] }\end{array}$ & Type & $\begin{array}{l}\text { Width } \\
\text { [min] }\end{array}$ & $\begin{array}{c}\text { Area } \\
{\left[\mathrm{mAU} \mathbf{U}^{\star} \mathrm{s}\right]}\end{array}$ & $\begin{array}{l}\text { Height } \\
\text { [mAU] }\end{array}$ & $\begin{array}{c}\text { Area } \\
8\end{array}$ \\
\hline$\frac{1}{2}$ & $\begin{array}{l}32.921 \\
40.188\end{array}$ & $\begin{array}{l}\text { BB } \\
\text { BB }\end{array}$ & $\begin{array}{l}1.1571 \\
1.4952\end{array}$ & $\begin{array}{l}1.31052 e^{4} \\
1.31341 e^{4}\end{array}$ & $\begin{array}{l}177.14226 \\
125: 26562\end{array}$ & $\begin{array}{l}49.9450 \\
50.0550\end{array}$ \\
\hline Total & : & & & $2.62393 e^{4}$ & 302.40788 & \\
\hline
\end{tabular}

Results obtained with enhanced integrator!

$\star \star \star$ End of Report $\star \star \star$

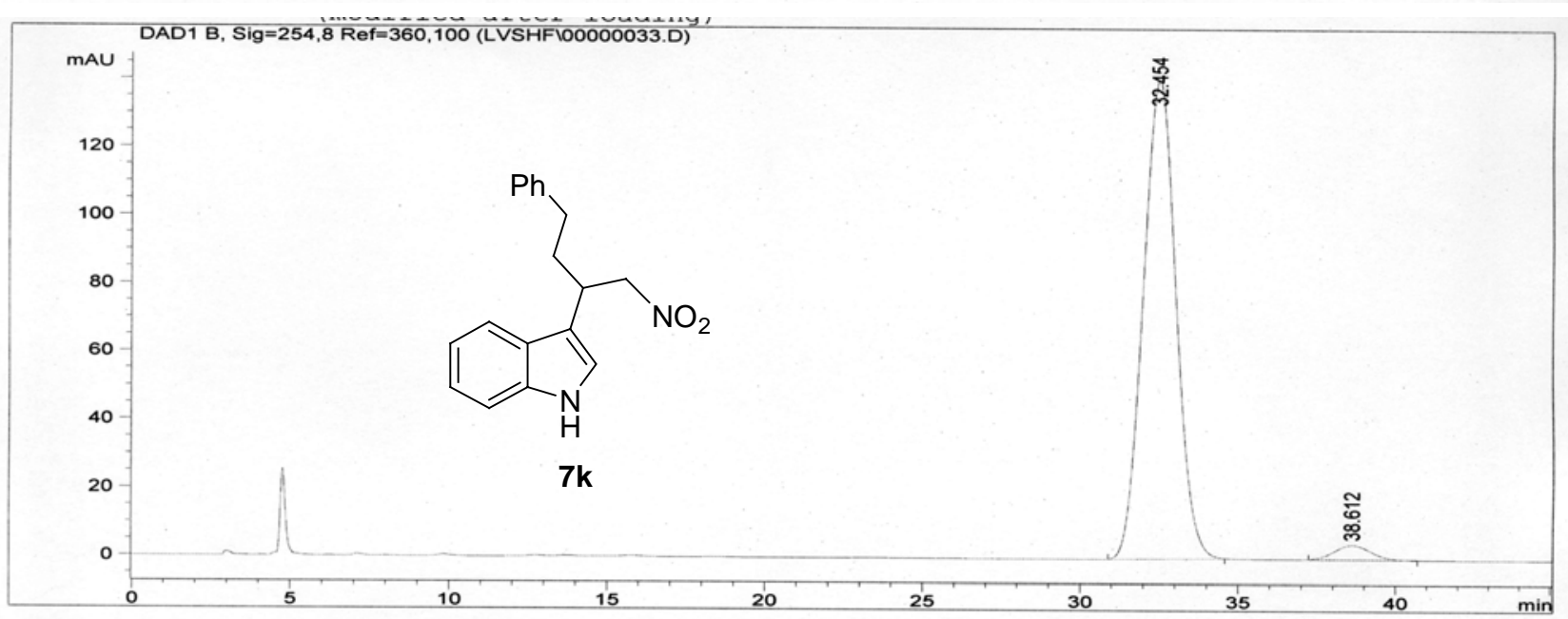

Area Percent Report

$\begin{array}{lll}\text { Sorted By } & : & \text { Signal } \\ \text { Multiplier } & : & 1.0000 \\ \text { Dilution } & : & 1.0000\end{array}$

Signal 1: DAD1 B, Sig=254, 8 Ref=360, 100

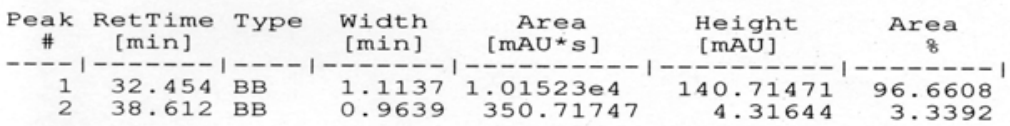

Totals : $1.05030 e^{4} 145.03115$

Results obtained with enhanced integrator!

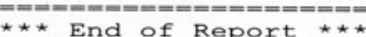



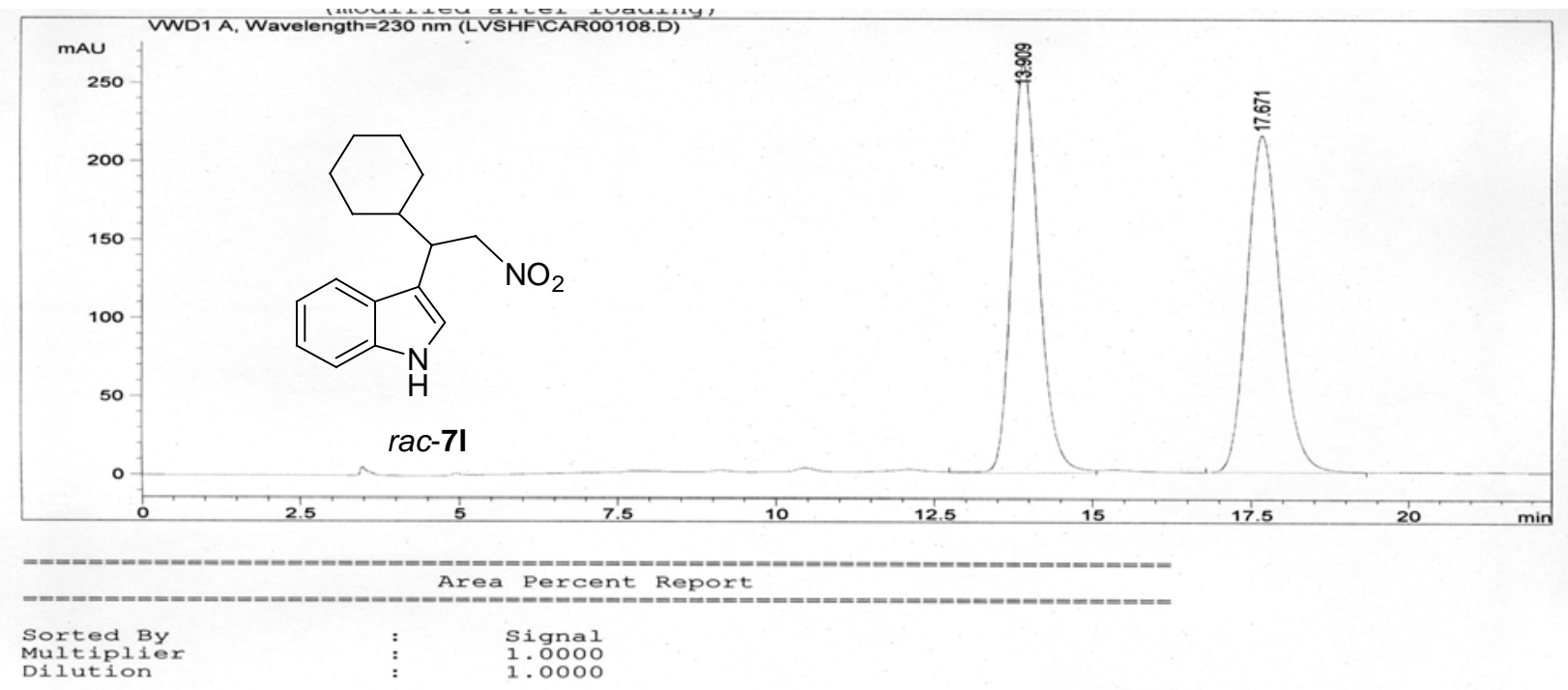

Signal 1: VWD1 A, wavelength-230 nm

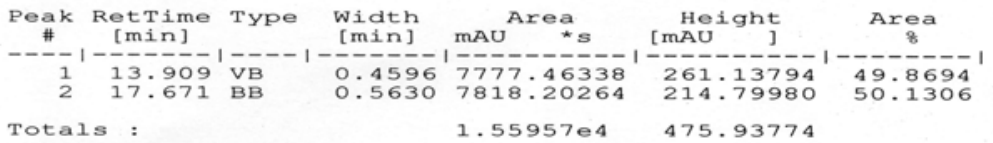

Results obtained with enhanced integrator!

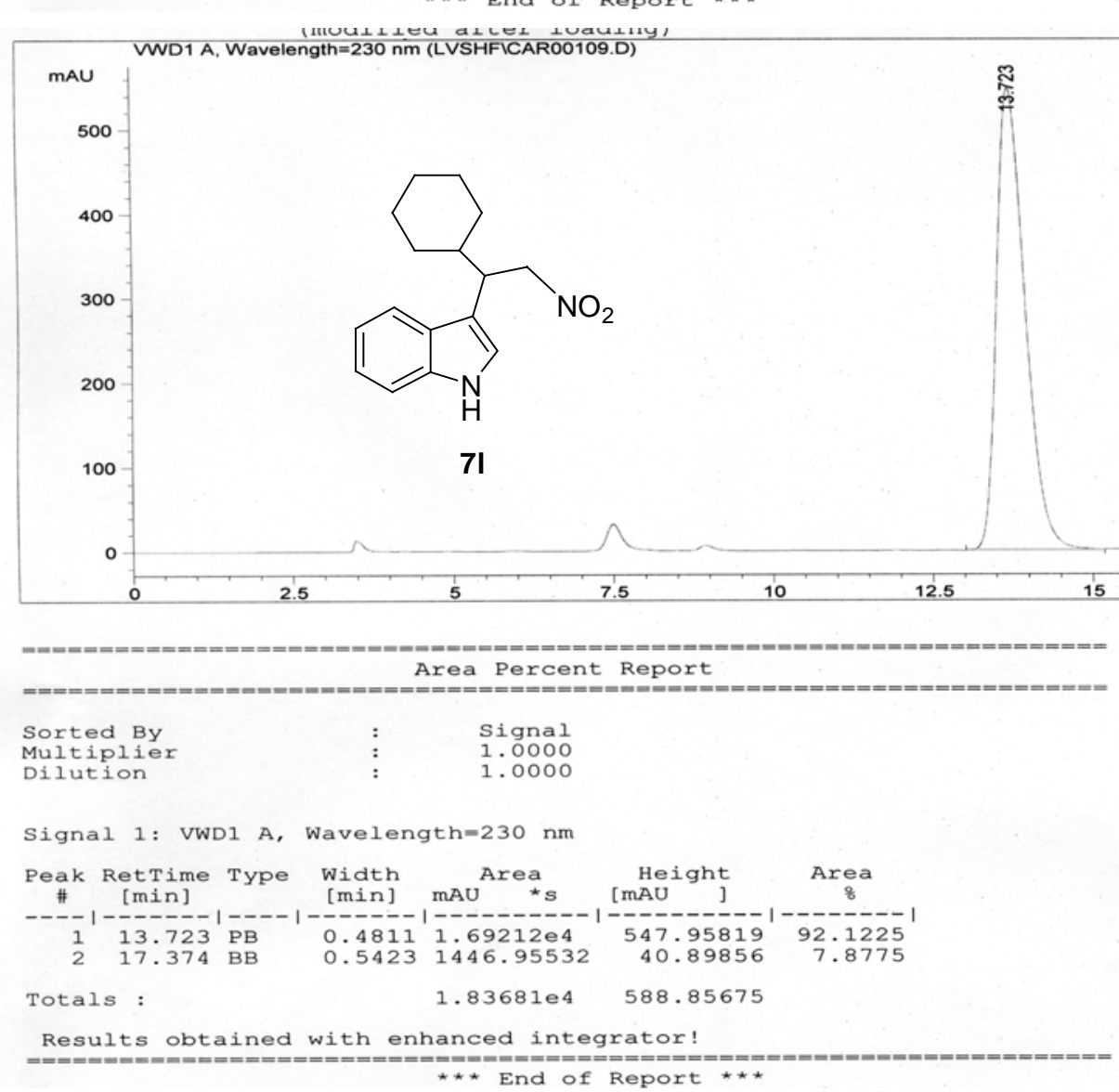




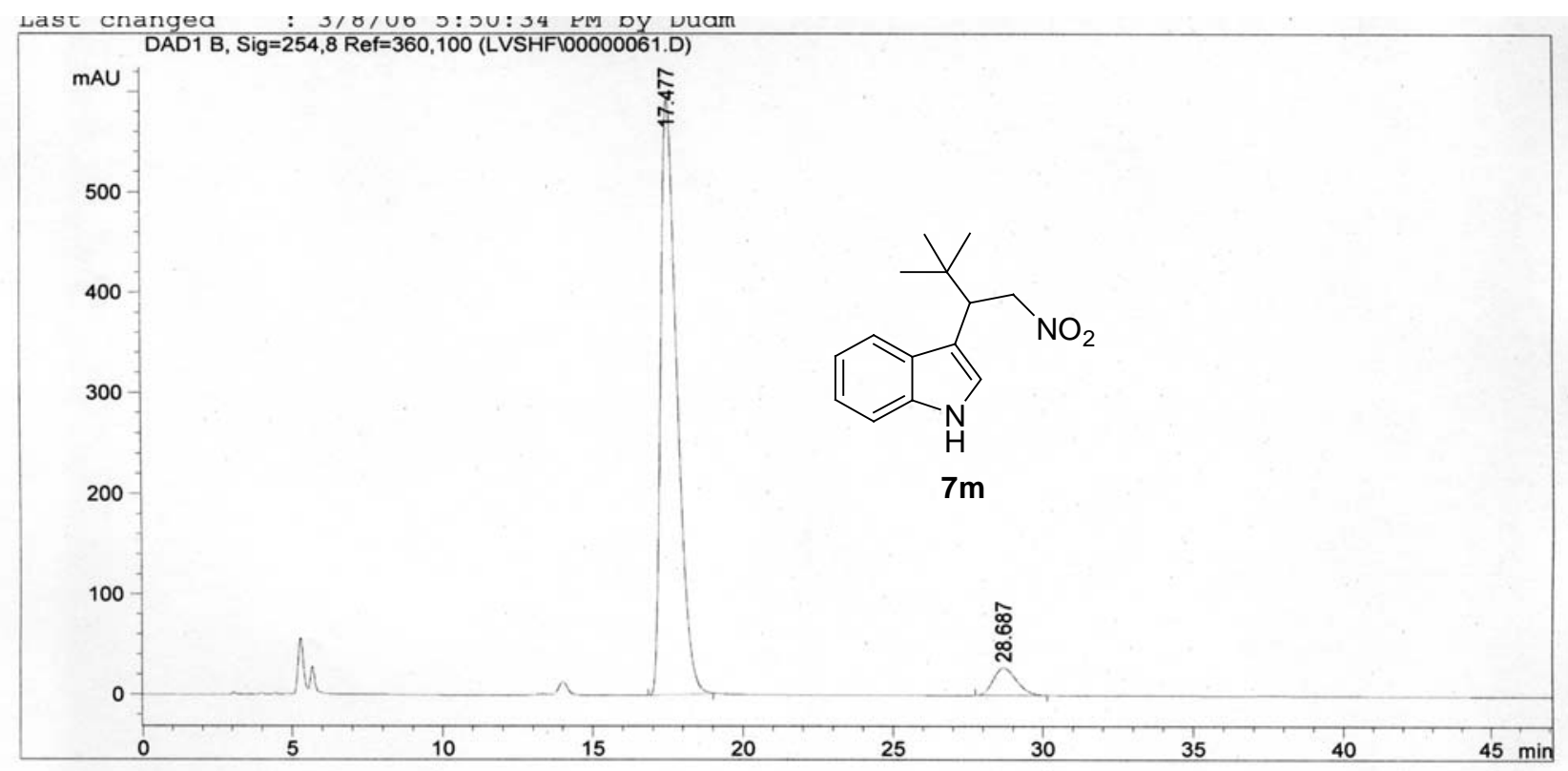

\section{Area Percent Report}

$\begin{array}{lll}\text { Sorted By } & : & \text { Signal } \\ \text { Multiplier } & : & 1.0000 \\ \text { Dilution } & : & 1.0000\end{array}$

Signal 1: DAD1 B, Sig=254, 8 Ref $=360,100$

\begin{tabular}{|c|c|c|c|c|c|c|}
\hline $\begin{array}{c}\text { Peak } \\
\#\end{array}$ & $\begin{array}{l}\text { RetTime } \\
\text { [min] }\end{array}$ & Type & $\begin{array}{l}\text { Width } \\
\text { [min] }\end{array}$ & $\begin{array}{c}\text { Area } \\
{\left[\mathrm{mAU}^{\star} \mathrm{s}\right]}\end{array}$ & $\begin{array}{l}\text { Height } \\
\text { [mAU] }\end{array}$ & $\underset{\frac{8}{8}}{\text { Area }}$ \\
\hline----1 & $--17---1$ & --1 & $-1--1$ & 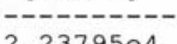 & 595 & ---1 \\
\hline $\begin{array}{l}1 \\
2\end{array}$ & $\begin{array}{l}17.477 \\
28.687\end{array}$ & $\begin{array}{l}\text { PB } \\
\text { BB }\end{array}$ & $\begin{array}{l}0.5724 \\
0.8577\end{array}$ & $\begin{array}{l}2.23795 \text { e } 4 \\
1509.12292\end{array}$ & $\begin{array}{r}595.49835 \\
27.52111\end{array}$ & $\begin{array}{r}93.6827 \\
6.3173\end{array}$ \\
\hline Total & : & & & $2.38886 e 4$ & 623.01946 & \\
\hline
\end{tabular}

Results obtained with enhanced integrator!

$\star \star \star$ End of Report $\star \star \star *$ 
Copies of ${ }^{1} \mathrm{H}$ NMR and ${ }^{13} \mathrm{C}$ NMR of nitroalkylated indoles $\mathbf{7 g}, \mathbf{7 j}, \mathbf{7 k}, \mathbf{7} \mathbf{l}$, and $\mathbf{7 m}$
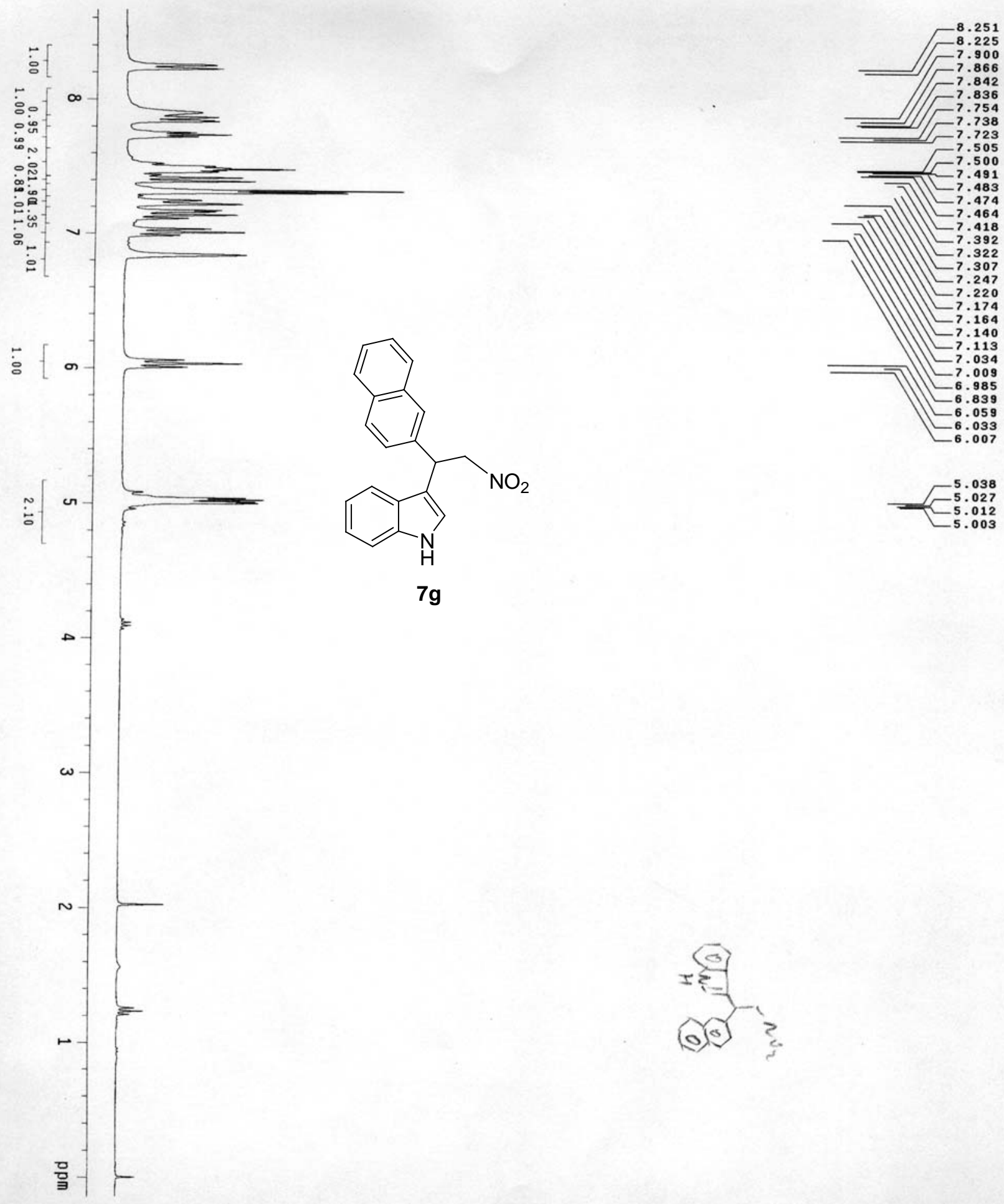


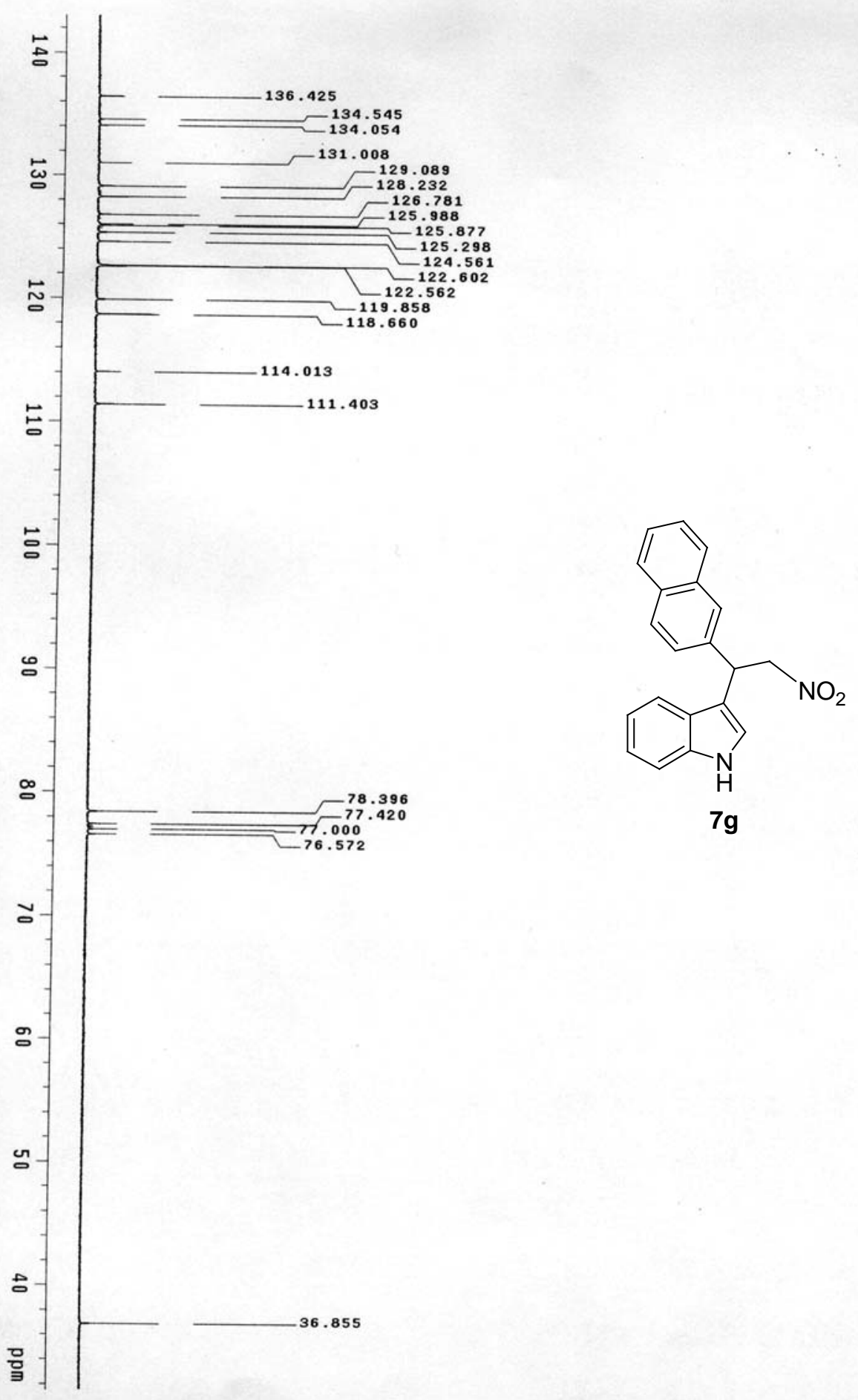




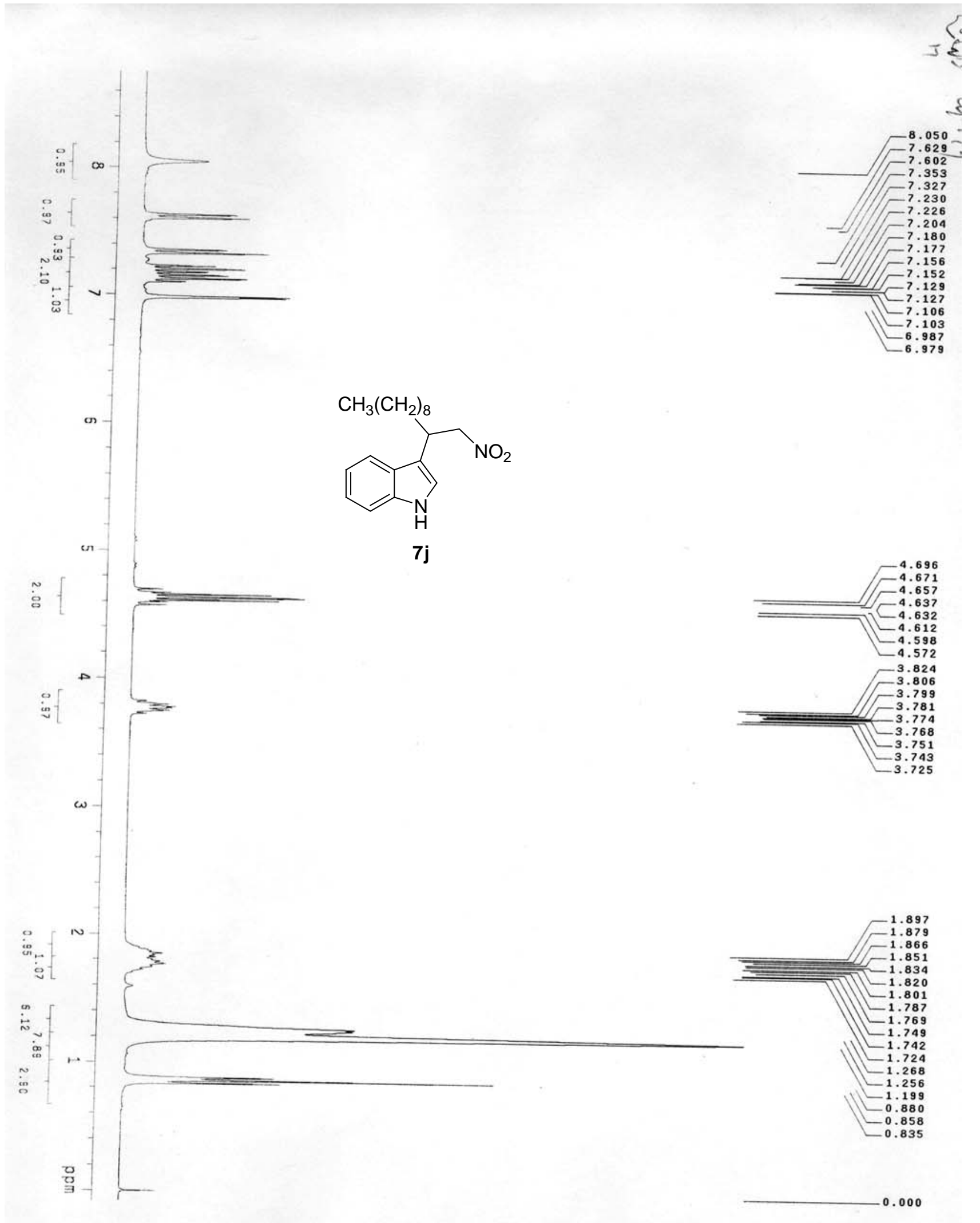




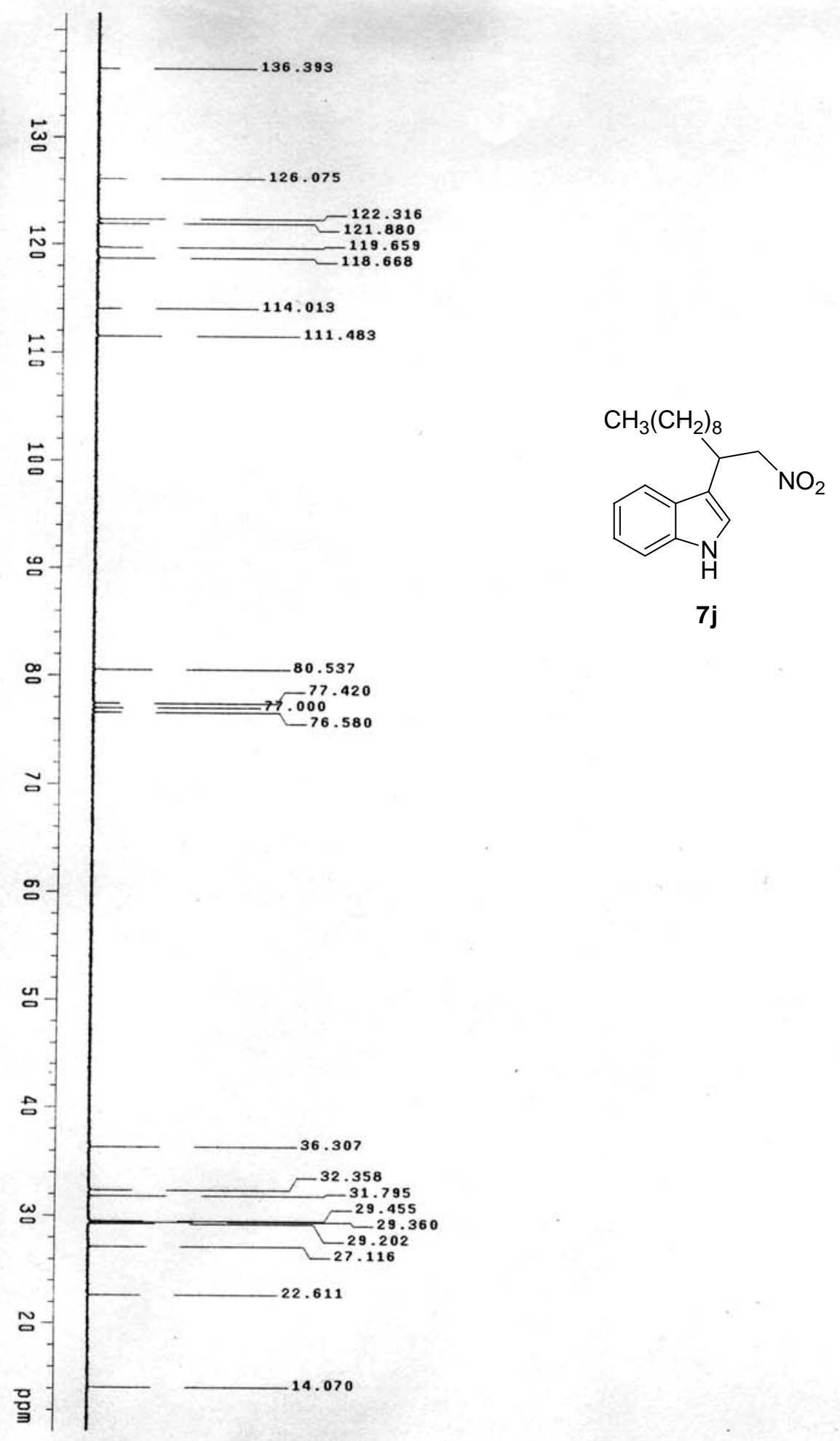



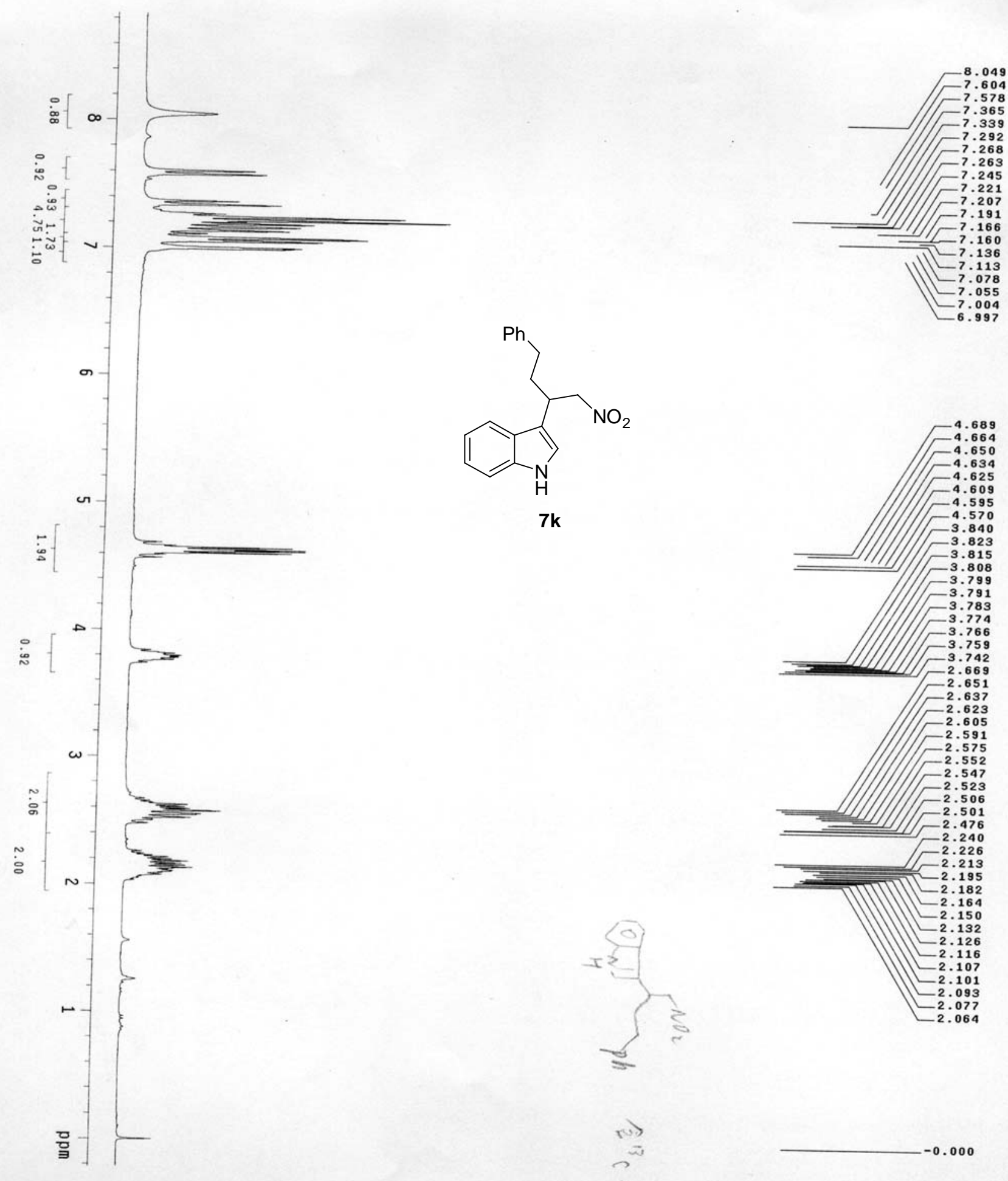

खो)

$-0.000$ 


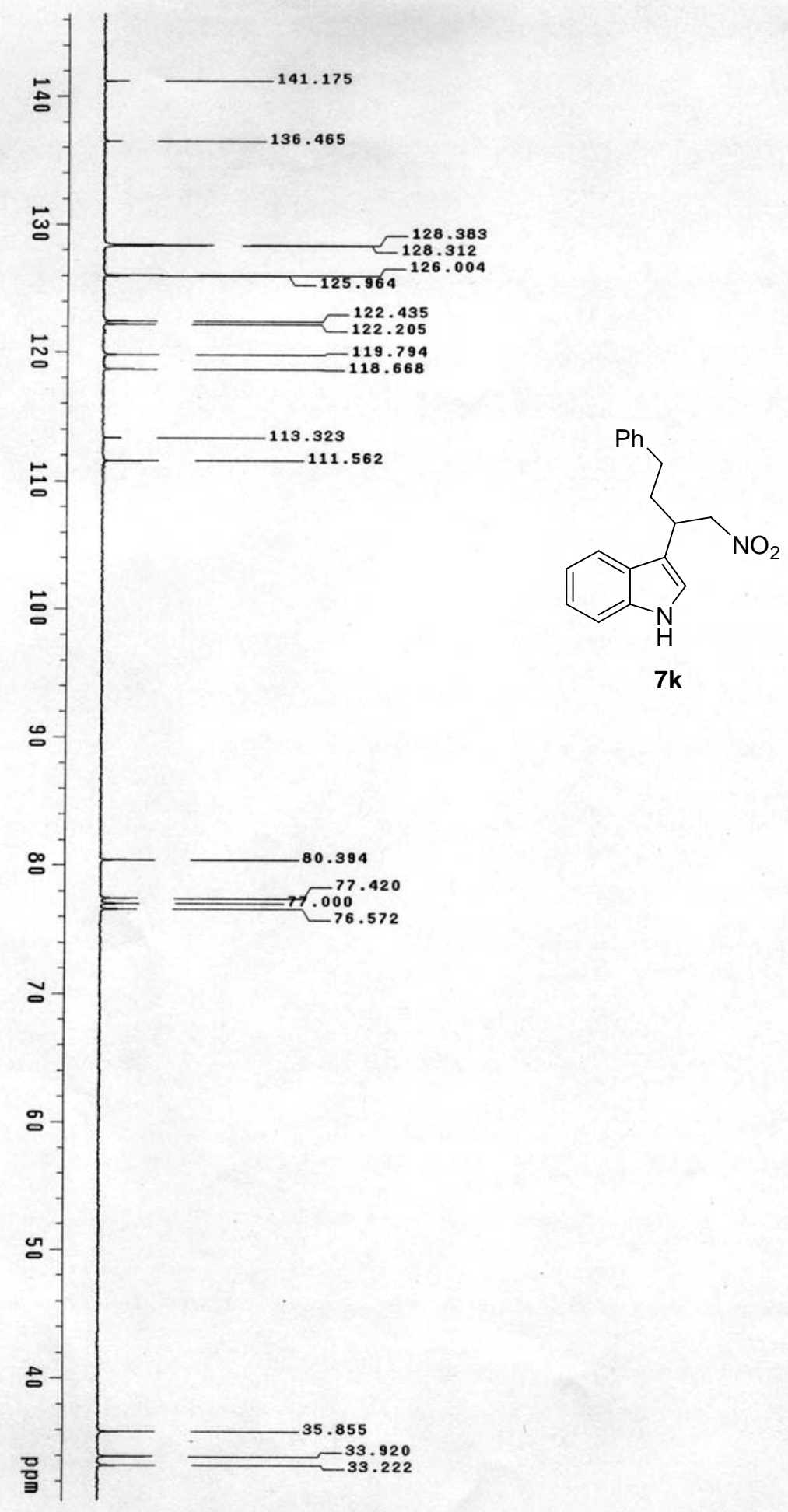




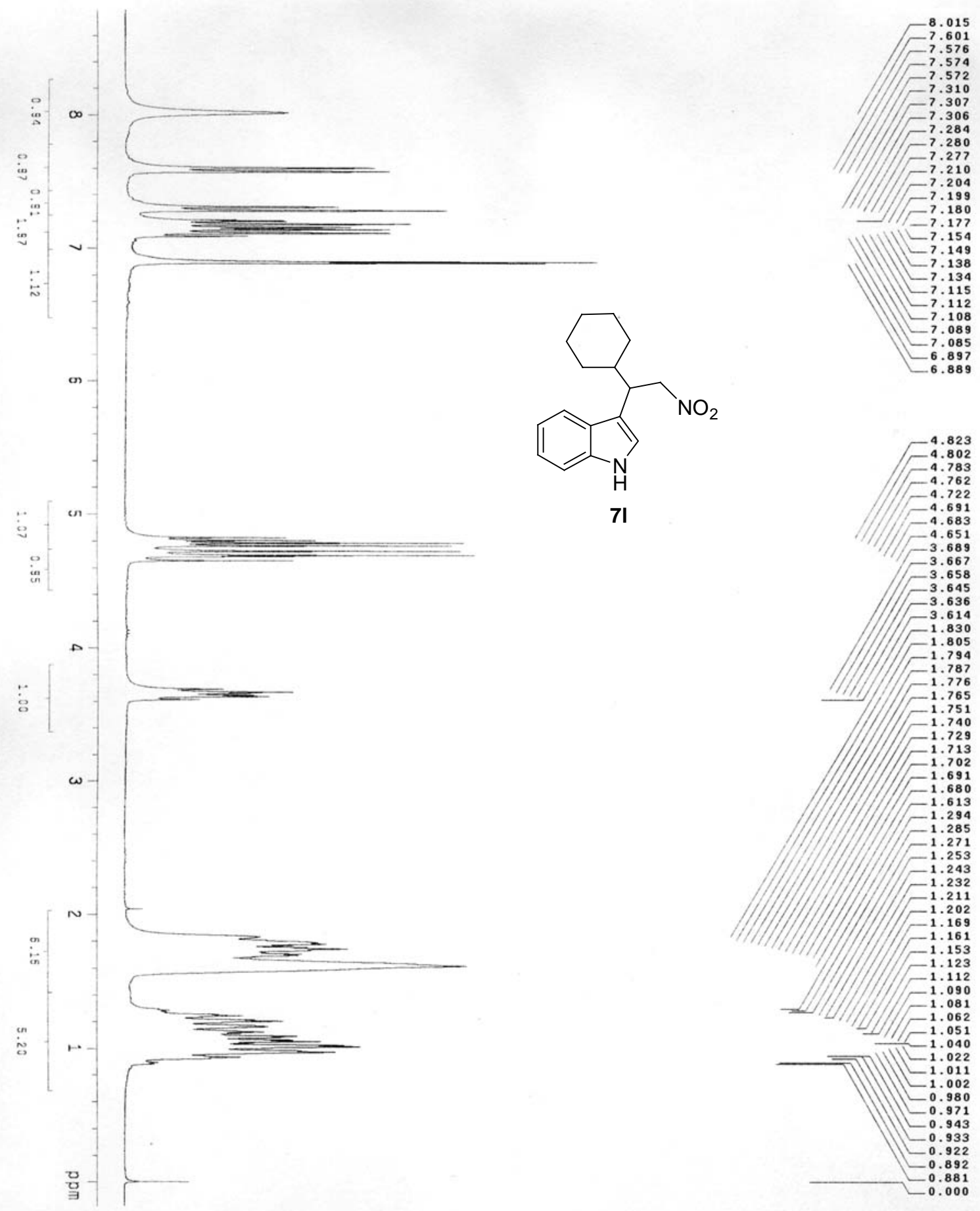




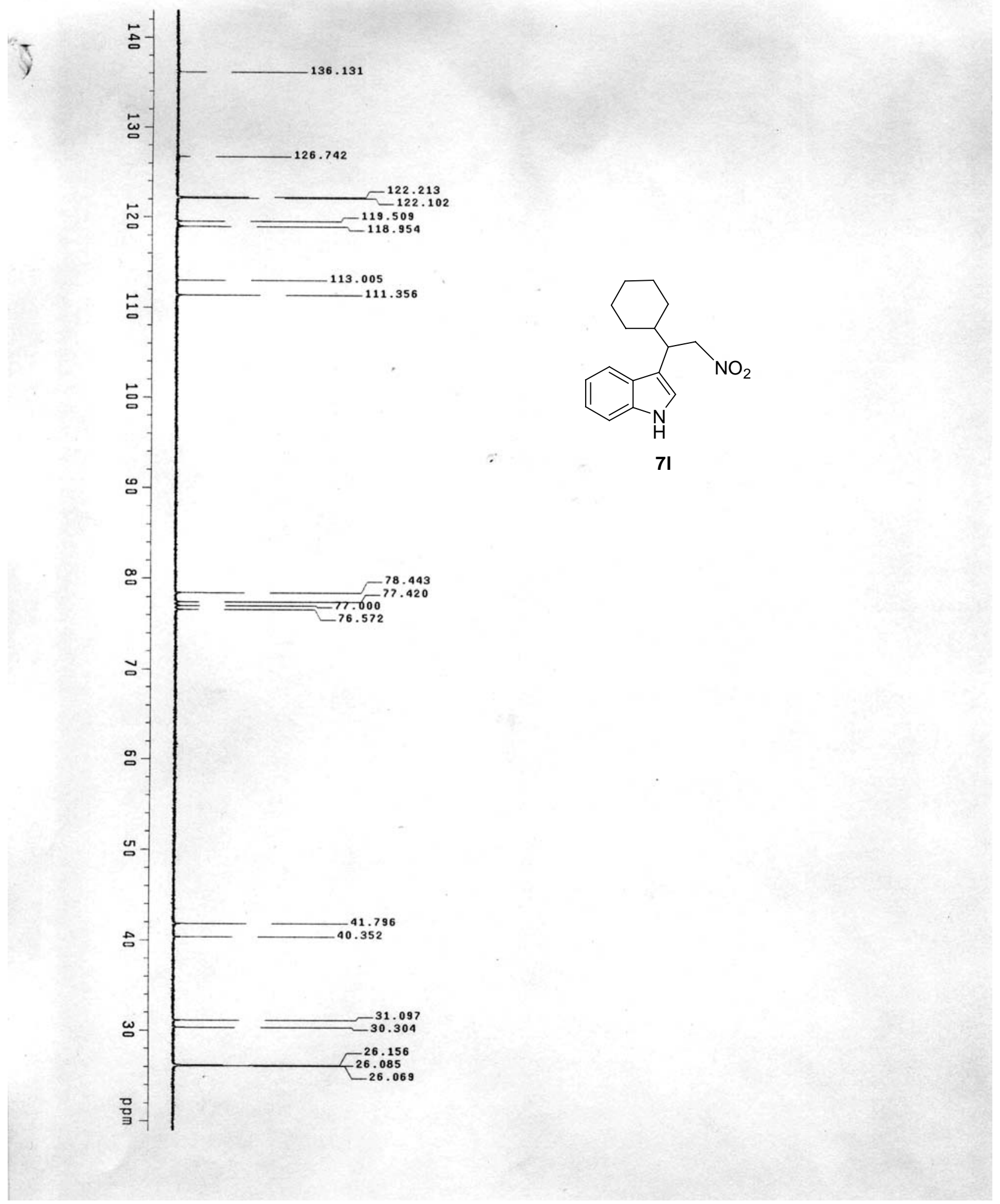




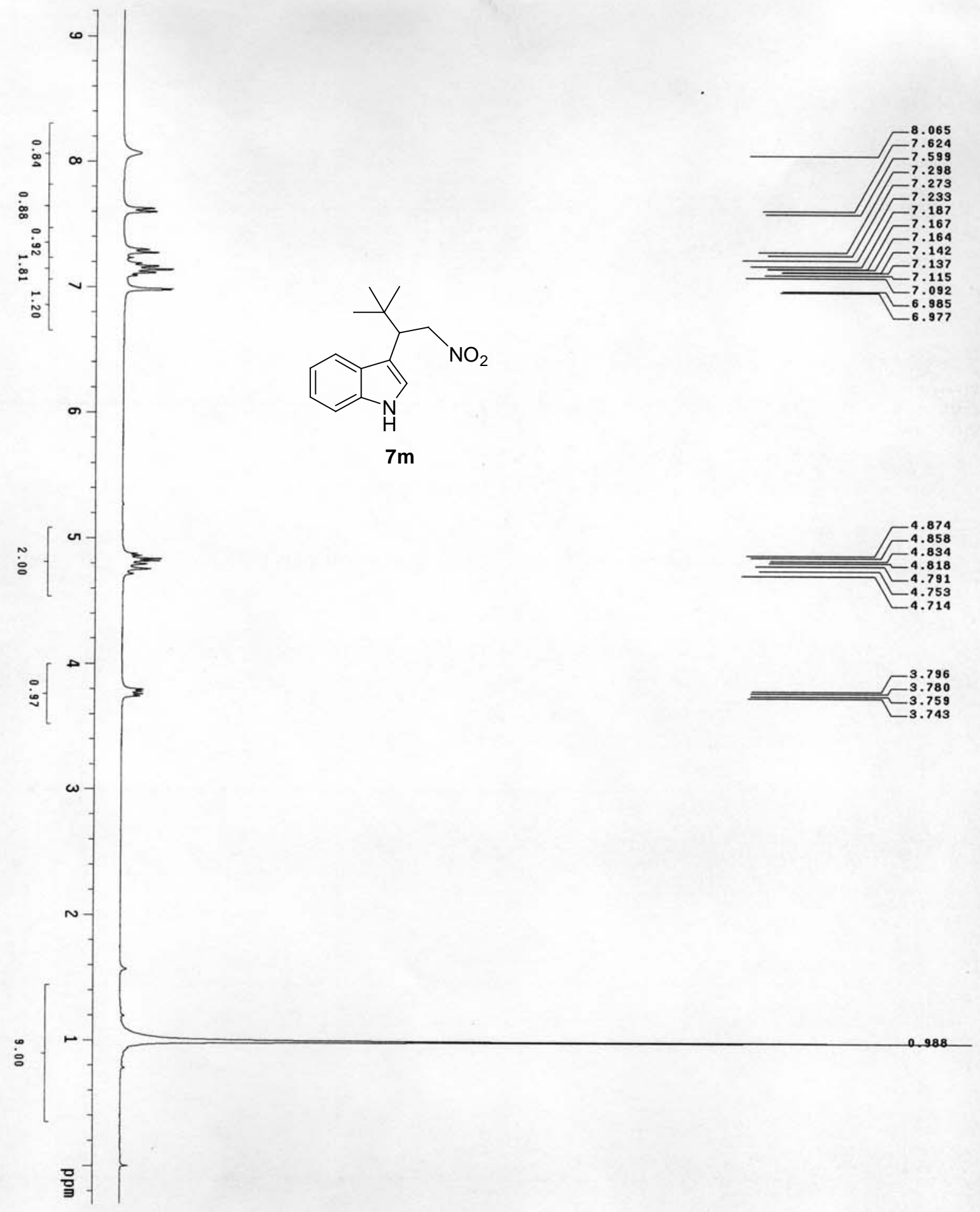

- S29 - 


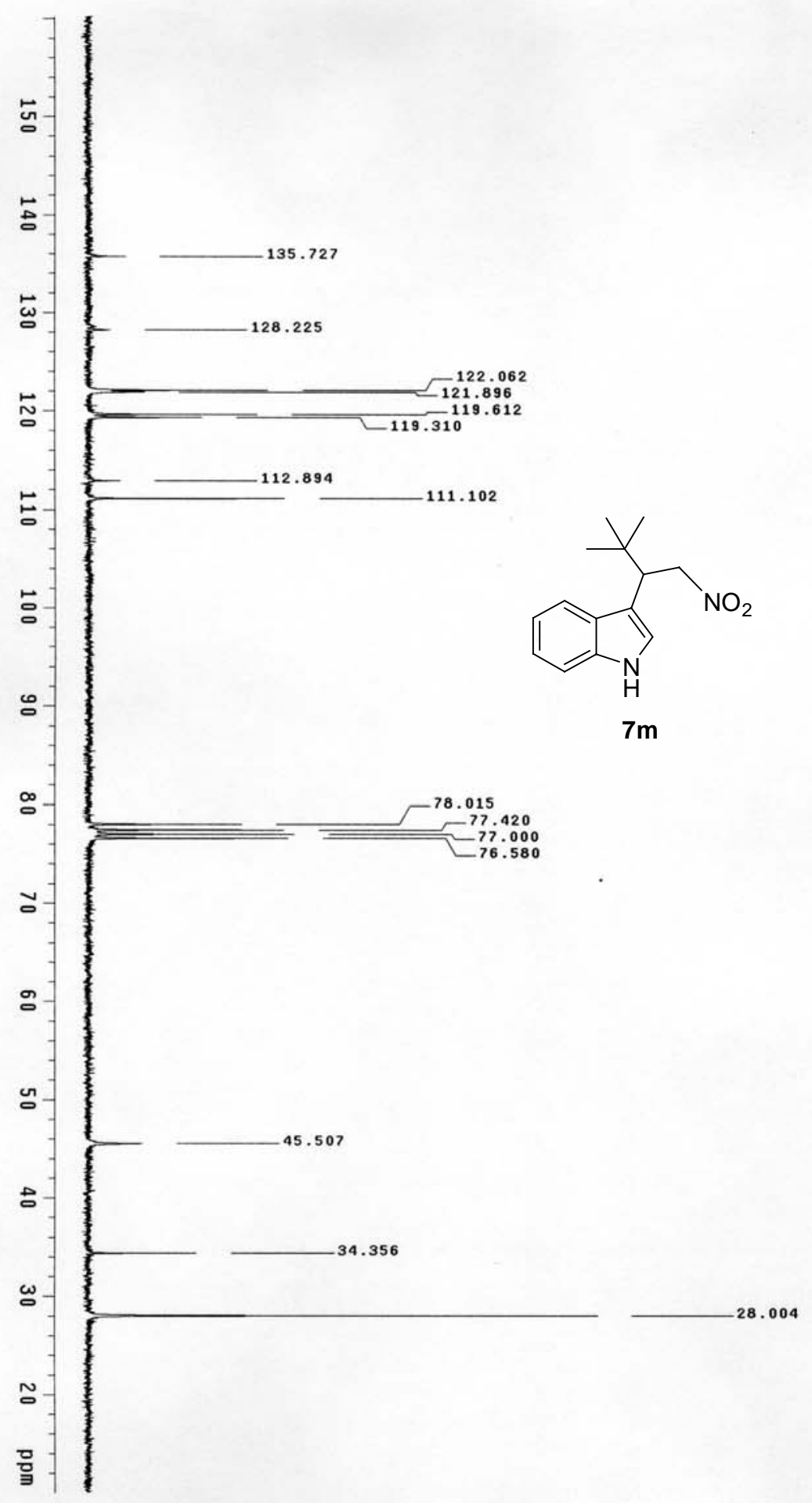

- S30 - 
Copies of ${ }^{1} \mathrm{H}$ NMR and ${ }^{13} \mathrm{C}$ NMR of ligand $\mathbf{4}$

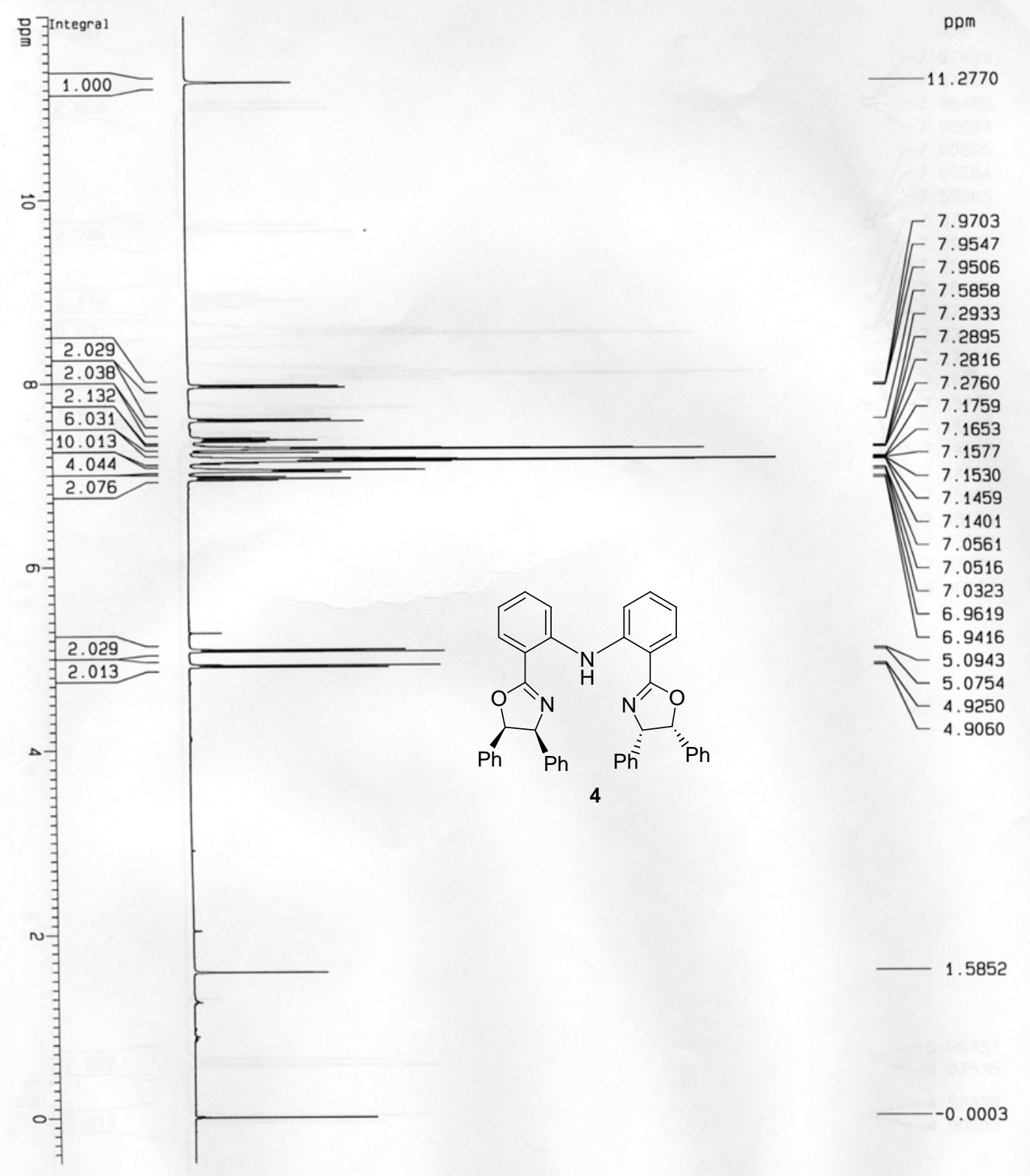

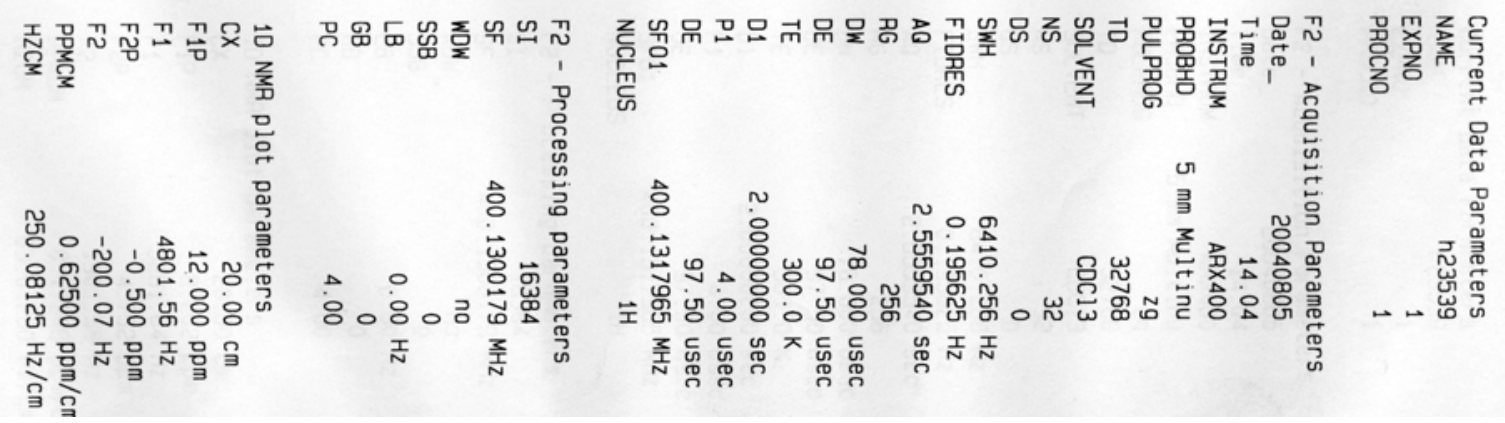




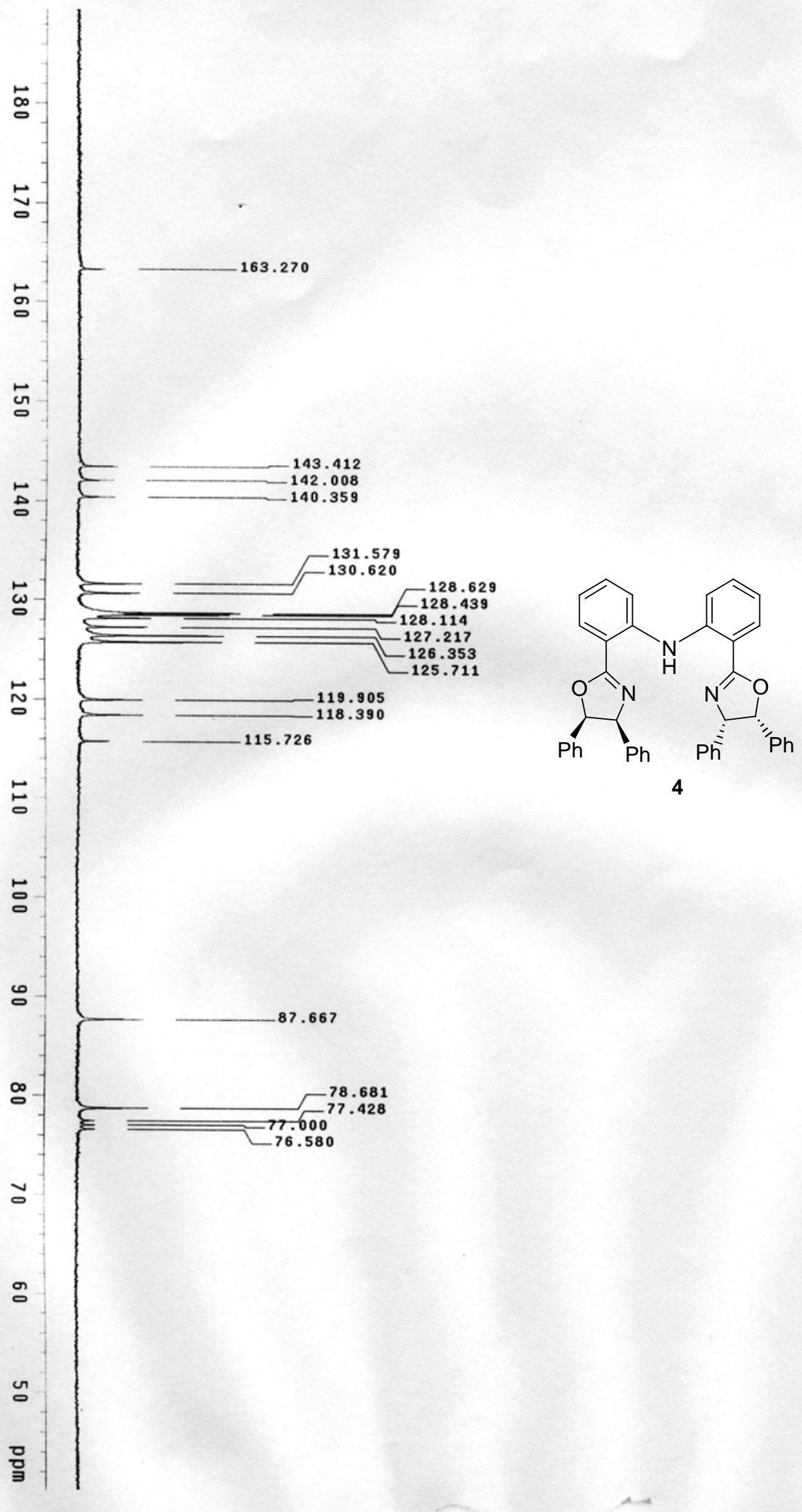

